TRANSACTIONS OF THE

AMERICAN MATHEMATICAL SOCIETY

Volume 362, Number 11, November 2010, Pages 5745-5770

S 0002-9947(2010)05101-3

Article electronically published on June 17, 2010

\title{
GROUPS OF VOLUME-PRESERVING DIFFEOMORPHISMS OF NONCOMPACT MANIFOLDS AND MASS FLOW TOWARD ENDS
}

\author{
TATSUHIKO YAGASAKI
}

\begin{abstract}
Suppose $M$ is a noncompact connected oriented $C^{\infty} n$-manifold and $\omega$ is a positive volume form on $M$. Let $\mathcal{D}^{+}(M)$ denote the group of orientation-preserving diffeomorphisms of $M$ endowed with the compact-open $C^{\infty}$ topology and let $\mathcal{D}(M ; \omega)$ denote the subgroup of $\omega$-preserving diffeomorphisms of $M$. In this paper we propose a unified approach for realization of mass transfer toward ends by diffeomorphisms of $M$. This argument, together with Moser's theorem, enables us to deduce two selection theorems for the groups $\mathcal{D}^{+}(M)$ and $\mathcal{D}(M ; \omega)$. The first one is the extension of Moser's theorem to noncompact manifolds, that is, the existence of sections for the orbit maps under the action of $\mathcal{D}^{+}(M)$ on the space of volume forms. This implies that $\mathcal{D}(M ; \omega)$ is a strong deformation retract of the group $\mathcal{D}^{+}\left(M ; E_{M}^{\omega}\right)$ consisting of $h \in \mathcal{D}^{+}(M)$, which preserves the set $E_{M}^{\omega}$ of $\omega$-finite ends of $M$.

The second one is related to the mass flow toward ends under volumepreserving diffeomorphisms of $M$. Let $\mathcal{D}_{E_{M}}(M ; \omega)$ denote the subgroup consisting of all $h \in \mathcal{D}(M ; \omega)$ which fix the ends $E_{M}$ of $M$. S. R. Alpern and V.S. Prasad introduced the topological vector space $\mathcal{S}(M ; \omega)$ of end charges of $M$ and the end charge homomorphism $c^{\omega}: \mathcal{D}_{E_{M}}(M ; \omega) \rightarrow \mathcal{S}(M ; \omega)$, which measures the mass flow toward ends induced by each $h \in \mathcal{D}_{E_{M}}(M ; \omega)$. We show that the homomorphism $c^{\omega}$ has a continuous section. This induces the factorization $\mathcal{D}_{E_{M}}(M ; \omega) \cong \operatorname{ker} c^{\omega} \times \mathcal{S}(M ; \omega)$, and it implies that $\operatorname{ker} c^{\omega}$ is a strong deformation retract of $\mathcal{D}_{E_{M}}(M ; \omega)$.
\end{abstract}

\section{INTRODUCTION}

The purpose of this article is to study topological properties of groups of volumepreserving diffeomorphisms of noncompact manifolds. The group of diffeomorphisms of a manifold $M$ acts on the space of volume forms on $M$. We can use a family of diffeomorphisms of $M$ to transfer volumes and thereby deform a family of volume forms on $M$. In this article we propose a unified approach for the general problem of realizing data of volume transfer toward ends by diffeomorphisms of $M$ (Theorem 3.1). We use this realization result, together with Moser's theorem, in order to obtain two main selection theorems related to volume-preserving diffeomorphisms of a noncompact manifold $M$ (Theorems [1.1] and [1.2) and deduce some conclusions on the group of volume-preserving diffeomorphisms of $M$ (Corollaries 1.1 and 1.2).

Received by the editors June 9, 2008.

2010 Mathematics Subject Classification. Primary 57S05, 58D05.

Key words and phrases. Group of volume-preserving diffeomorphisms, mass flow, end charge homomorphism, $\sigma$-compact manifold.

(C)2010 American Mathematical Society Reverts to public domain 28 years from publication 
Suppose $M$ is a connected oriented separable metrizable smooth $n$-manifold possibly with boundary and $\omega$ is a positive volume form on $M$. Let $\mathcal{D}^{+}(M)$ denote the group of orientation-preserving diffeomorphisms of $M$ endowed with the compactopen $C^{\infty}$-topology and let $\mathcal{D}(M ; \omega)$ denote the subgroup consisting of $\omega$-preserving diffeomorphisms of $M$.

Our first concern is the relation between the groups $\mathcal{D}^{+}(M) \supset \mathcal{D}(M ; \omega)$. Let $\mathcal{V}^{+}(M)$ denote the space of positive volume forms $\mu$ on $M$ endowed with the compact-open $C^{\infty}$-topology, and for $m \in(0, \infty]$ let $\mathcal{V}^{+}(M, m)=\left\{\mu \in \mathcal{V}^{+}(M) \mid\right.$ $\mu(M)=m\}$. The group $\mathcal{D}^{+}(M)$ acts continuously on $\mathcal{V}^{+}(M, m)$ by $h \cdot \mu=h_{*} \mu(=$ $\left.\left(h^{-1}\right)^{*} \mu\right)$, and the group $\mathcal{D}(M ; \omega)$ coincides with the stabilizer of $\omega$ under this action.

When $M$ is compact, Moser's theorem [2, 3, 9, asserts the transitivity and the existence of sections of the orbit maps under this action. Similar results for measurepreserving homeomorphisms were obtained by von Neumann-Oxtoby-Ulam [11] and A. Fathi [7].

When $M$ is noncompact, it is necessary to include some information on the ends of $M$ [4, 8]. For an open subset $F$ of the space $E_{M}$ of the ends of $M$, consider the subgroup $\mathcal{D}^{+}(M ; F)=\left\{h \in \mathcal{D}^{+}(M) \mid \bar{h}(F)=F\right\}$, where $\bar{h}$ is the natural homeomorphic extension of $h$ to the end compactification of $M$. We also need to introduce the spaces

$$
\mathcal{V}^{+}(M ; F)_{e w}=\left\{\mu \in \mathcal{V}^{+}(M) \mid E_{M}^{\mu}=F\right\}
$$

and

$$
\mathcal{V}^{+}(M ; m, F)_{e w}=\mathcal{V}^{+}(M ; F) \cap \mathcal{V}^{+}(M ; m)
$$

where $E_{M}^{\mu}$ is the set of $\mu$-finite ends of $M$. According to R. Berlanga 4 these spaces are endowed with the finite-ends weak $C^{\infty}$-topology ew (cf. Section 2.4). The group $\mathcal{D}^{+}(M ; F)$ acts continuously on $\mathcal{V}^{+}(M ; m, F)_{e w}$ by $h \cdot \mu=h_{*} \mu$, and the subgroup $\mathcal{D}(M ; \omega)$ coincides with the stabilizer of $\omega$ under this action. The transitivity of this action was shown by R. E. Greene and K. Shiohama 8 . A similar problem for measure-preserving homeomorphisms was studied by R. Belranga [5]. He has really obtained the result on the existence of sections for orbit maps 4 . The first application of Theorem 3.1 is the $C^{\infty}$-version of this result, that is, the extension of Moser's theorem to noncompact manifolds. Let $\mathcal{D}_{\partial}(M)_{1}$ denote the path-component of $i d_{M}$ in the group $\mathcal{D}_{\partial}(M)=\left\{h \in \mathcal{D}(M)|h|_{\partial M}=i d_{\partial M}\right\}$.

Theorem 1.1. Suppose $P$ is any topological space and $\mu, \nu: P \rightarrow \mathcal{V}^{+}(M ; F)_{\text {ew }}$ are continuous maps with $\mu_{p}(M)=\nu_{p}(M)(p \in P)$. Then there exists a continuous map $h: P \rightarrow \mathcal{D}_{\partial}(M)_{1}$ such that for each $p \in P$

(1) $h_{p_{*}} \mu_{p}=\nu_{p}$,

(2) if $\mu_{p}=\nu_{p}$, then $h_{p}=i d_{M}$.

Corollary 1.1. Let $\omega \in \mathcal{V}^{+}(M)$ and set $m=\omega(M)$ and $F=E_{M}^{\omega}$.

(1) The orbit map $\pi_{\omega}: \mathcal{D}^{+}(M ; F) \rightarrow \mathcal{V}^{+}(M ; m, F)_{\text {ew }}, \pi_{\omega}(h)=h_{*} \omega$, admits a continuous section $\sigma: \mathcal{V}^{+}(M ; m, F)_{\text {ew }} \rightarrow \mathcal{D}_{\partial}(M)_{1}$ such that $\sigma(\omega)=i d_{M}$.

(2) (i) $\mathcal{D}^{+}(M ; F) \cong \mathcal{V}^{+}(M ; m, F)_{e w} \times \mathcal{D}(M ; \omega)$.

(ii) $\mathcal{D}(M ; \omega)$ is a strong deformation retract of $\mathcal{D}^{+}(M ; F)$.

Next we are concerned with an internal structure of the group $\mathcal{D}(M ; \omega)$. When $M$ is noncompact, one can measure volume transfer toward ends of $M$ under volumepreserving diffeomorphisms which fix the ends $E_{M}$. This quantity is described by 
the end charge homomorphism

$$
c^{\omega}: \mathcal{D}_{E_{M}}(M ; \omega) \rightarrow \mathcal{S}(M ; \omega)
$$

introduced by S. R. Alpern and V.S. Prasad [1. An end charge of $M$ is a finitely additive signed measure on the algebra of clopen subsets of $E_{M}$. Let $\mathcal{S}(M)$ denote the topological linear space of all end charges of $M$ with the weak topology, and let $\mathcal{S}(M ; \omega)$ denote the linear subspace of $\mathcal{S}(M)$ consisting of end charges $c$ of $M$ with $c\left(E_{M}\right)=0$ and $\left.c\right|_{E_{M}^{\omega}}=0$. For each $h \in \mathcal{D}_{E_{M}}(M ; \omega)$ an end charge $c_{h}^{\omega} \in \mathcal{S}(M ; \omega)$ is defined by

$$
c_{h}^{\omega}\left(E_{C}\right)=\omega(C-h(C))-\omega(h(C)-C),
$$

where $C$ is any $n$-submanifold of $M$ such that $\operatorname{Fr}_{M} C$ is compact and $E_{C} \subset E_{M}$ is the set of ends of $C$. This quantity represents the total $\omega$-volume (or mass) transferred by $h$ into $C$ and into $E_{C}$ in the last. Hence, the end charge $c_{h}^{\omega}$ describes mass flow toward ends induced by $h$.

The group $\mathcal{D}_{E_{M}}(M ; \omega)$ acts continuously on $\mathcal{S}(M ; \omega)$ by $h \cdot a=c_{h}^{\omega}+a$. The end charge homomorphism $c^{\omega}: \mathcal{D}_{E_{M}}(M ; \omega) \rightarrow S(M ; \omega)$ coincides with the orbit map at $0 \in \mathcal{S}(M ; \omega)$ under this action. In [12] we have shown that the end charge homomorphism for measure-preserving homeomorphisms has a continuous section. As the second application of Theorem 3.1 we show that the end charge homomorphism $c^{\omega}$ for volume-preserving diffeomorphisms also has a continuous (nonhomomorphic) section.

Theorem 1.2. Suppose $P$ is any topological space and $\mu: P \rightarrow \mathcal{V}^{+}(M)$ and $a: P \rightarrow \mathcal{S}(M)$ are continuous maps such that $a_{p} \in \mathcal{S}\left(M ; \mu_{p}\right)(p \in P)$. Then there exists a continuous map $h: P \rightarrow \mathcal{D}_{\partial}(M)_{1}$ such that for each $p \in P$,

(1) $h_{p} \in \mathcal{D}_{\partial}\left(M ; \mu_{p}\right)_{1}$,

(2) $c_{h_{p}}^{\mu_{p}}=a_{p}$,

(3) if $a_{p}=0$, then $h_{p}=i d_{M}$.

Corollary 1.2. Let $\omega \in \mathcal{V}^{+}(M)$.

(1) The end charge homomorphism $c^{\omega}: \mathcal{D}_{E_{M}}(M ; \omega) \rightarrow \mathcal{S}(M ; \omega)$ admits a continuous section $s: \mathcal{S}(M ; \omega) \rightarrow \mathcal{D}_{\partial}(M ; \omega)_{1}$ such that $s(0)=i d_{M}$.

(2) (i) $\mathcal{D}_{E_{M}}(M ; \omega) \cong \operatorname{ker} c^{\omega} \times \mathcal{S}(M ; \omega)$.

(ii) $\operatorname{ker} c^{\omega}$ is a strong deformation retract of $\mathcal{D}_{E_{M}}(M ; \omega)$.

The group $\operatorname{ker} c^{\omega}$ contains the subgroup $\mathcal{D}^{c}(M ; \omega)$ consisting of $\omega$-preserving diffeomorphisms with compact support. In a succeeding paper we will investigate the relation between these subgroups.

This paper is organized as follows. Section 2 is devoted to generalities on end compactifications, diffeomorphism groups, spaces of volume forms and end charge homomorphism. In Sections 3.1 and 3.2 we discuss volume transfer by engulfing isotopies and we obtain a fundamental deformation lemma. In Section 3.4 we state and prove Theorem 3.1 the main theorem in this article. Theorems 1.1, 1.2 and their corollaries are proved in Section 4.

\section{Preliminaries}

2.1. Conventions. Throughout the paper, a $C^{\infty} n$-manifold $M$ means a separable metrizable $C^{\infty} n$-manifold, possibly with boundary. We add the phrase "possibly with corners" when we allow $M$ to have corners modeled on $[0, \infty)^{2} \times \mathbb{R}^{n-2}$. In this 
paper corners appear only when we cut an $n$-manifold with boundary by a proper $(n-1)$-submanifold.

Suppose $M$ is a $C^{\infty} n$-manifold, possibly with corners. The notation Int $M$ and $\partial=\partial M$ denote the interior and boundary of $M$ as a manifold. For a subset $A$ of $M$, let $\operatorname{Fr}_{M} A, \operatorname{cl}_{M} A$ and $\operatorname{Int}_{M} A$ denote the frontier, closure and interior of $A$ relative to $M$.

A $C^{\infty} n$-submanifold $N$ of $M$ means a closed subset of $M$ such that (a) $\operatorname{Fr}_{M} N$ is a proper $C^{\infty}(n-1)$-submanifold of $M$ and (b) $\operatorname{Fr}_{M} N$ does not meet the corners of $M$. Hence, $N$ is a $C^{\infty} n$-manifold with corners $\operatorname{Fr}_{M} N \cap \partial M$ (together with other corners of $M$ included in $N)$ and $N^{c} \equiv c l_{M}(M-N)$ is also a $C^{\infty} n$-submanifold of $M$.

When $M$ has corners, a collar $E$ of $\partial M$ in $M$ is locally modeled on $E_{0} \times \mathbb{R}^{n-2}$, where $E_{0}=[0,2]^{2} \backslash[0,1)^{2} \subset \mathbb{R}^{2}$. The collar $E_{0}$ has the base $C=[0,2]^{2} \backslash[0,2)^{2}$ and the collar arcs $I_{x}=\{(1-t / 2) x \mid t \in[0,1]\}(x \in C)$ (so that the level sets consist of $\left.F_{t}=(1-t / 2) C(t \in[0,1])\right)$. The notation $E=\partial M \times[0,1]$ simply indicates the (nondiffeomorphic) parametrization of $E$.

The symbols $\mathcal{B}(M), \mathcal{K}(M)$ and $\mathcal{C}(M)$ denote the sets of Borel subsets, compact subsets and connected components of $M$, respectively. Let $\mathcal{B}_{c}(M)=\{C \in \mathcal{B}(M) \mid$ $\operatorname{Fr}_{M} C$ : compact $\}$ and $\mathcal{B}_{c, 0}(M)=\left\{C \in \mathcal{B}_{c}(M) \mid \operatorname{Fr}_{M} C\right.$ has zero Lebesgue measure $\}$.

A Radon measure on $M$ is a Borel measure $\mu$ on $M$ such that $\mu(K)<\infty$ for each $K \in \mathcal{K}(M)$. A Radon measure $\mu$ on $M$ is called good if $\mu(p)=0$ for each point $p \in M$ and $\mu(U)>0$ for any nonempty open subset $U$ of $M$. Let $\mathcal{M}_{g}^{\partial}(M)$ denote the space of good Radon measures $\mu$ on $M$ with $\mu(\partial M)=0$ endowed with the weak topology. For $\mu, \nu \in \mathcal{M}_{g}^{\partial}(M)$ we say that $\nu$ is $\mu$-biregular if $\mu$ and $\nu$ have the same collection of null sets. Let $\mathcal{M}_{g}^{\partial}(M, \widehat{\mu}$-reg $)=\left\{\nu \in \mathcal{M}_{g}^{\partial}(M) \mid \nu\right.$ is $\mu$-biregular $\}$ (cf. [1, 4, 7, 12]).

By $C^{\infty}(M)$ we denote the space of $C^{\infty}$-functions $f: M \rightarrow \mathbb{R}$ endowed with the compact-open $C^{\infty}$-topology. For $I \subset \mathbb{R}$ we have the subspace $C^{\infty}(M, I)=\{f \in$ $\left.C^{\infty}(M) \mid f(M) \subset I\right\}$.

The symbol $\Omega^{n}(M)$ denotes the space of $n$-forms on $M$ endowed with the compact-open $C^{\infty}$-topology. When $M$ is oriented, $\mathcal{V}^{+}(M)$ denotes the subspace of $\Omega^{n}(M)$ consisting of positive volume forms on $M$. For any $\omega \in \mathcal{V}^{+}(M)$, the space $\mathcal{V}^{+}(M)$ admits a canonical affine contraction onto $\{\omega\}$ :

$$
\varphi: \mathcal{V}^{+}(M) \times[0,1] \rightarrow \mathcal{V}^{+}(M), \varphi_{t}(\mu)=(1-t) \mu+t \omega
$$

Each $\omega \in \mathcal{V}^{+}(M)$ induces a Radon measure $\widehat{\omega} \in \mathcal{M}_{g}^{\partial}(M)$ defined by

$$
\widehat{\omega}(B)=\int_{B} \omega \equiv \int_{M} \chi_{B} \omega \quad(B \in \mathcal{B}(M)),
$$

where $\chi_{B}$ is the characteristic function of $B$ (i.e., $\chi_{B} \equiv 1$ on $B$ and $\chi_{B} \equiv 0$ on $M-B)$. Since $\widehat{\mu} \in \mathcal{M}_{g}^{\partial}(M, \widehat{\omega}-\mathrm{reg})$ for any $\mu \in \mathcal{V}^{+}(M)$, this determines a canonical continuous map $\mathcal{V}^{+}(M) \rightarrow \mathcal{M}_{g}^{\partial}(M, \omega$-reg). For simplicity, we denote $\widehat{\omega}$ by the same symbol $\omega$. Note that $C \in \mathcal{B}(M)$ has zero Lebesgue measure iff $\omega(C)=0$.

By $\mathcal{D}(M)$ we denote the group of diffeomorphisms of $M$ onto itself endowed with the compact-open $C^{\infty}$-topology. The support of $h \in \mathcal{D}(M)$ is defined by $\operatorname{supp} h=\operatorname{cl}_{M}\{x \in M \mid h(x) \neq x\}$. Let $\mathcal{D}^{c}(M)=\{h \in \mathcal{D}(M) \mid \operatorname{supp} h$ : compact $\}$, and for $A, B \subset M$ we set

$$
\mathcal{D}_{A}(M, B)=\left\{h \in \mathcal{D}(M)|h|_{A}=i d_{A}, h(B)=B\right\}
$$


and

$$
\mathcal{D}_{A}^{c}(M, B)=\mathcal{D}_{A}(M, B) \cap \mathcal{D}^{c}(M) .
$$

When $M$ is oriented, $\mathcal{D}^{+}(M)$ denotes the subgroup of $\mathcal{D}(M)$ consisting of orientation-preserving diffeomorphisms of $M$. For $\omega \in \mathcal{V}^{+}(M)$ we say that $h \in \mathcal{D}(M)$ preserves $\omega$ if the pullback $h^{*} \omega=\omega$ (or the pushforward $h_{*} \omega \equiv\left(h^{-1}\right)^{*} \omega=\omega$ ). Let $\mathcal{D}(M ; \omega)$ denote the subgroup of $\mathcal{D}^{+}(M)$ consisting of $\omega$-preserving diffeomorphisms of $M$.

For any subgroup $G$ of $\mathcal{D}(M)$, the symbol $G_{1}$ denotes the path-component of $i d_{M}$ in $G$. When $G \subset \mathcal{D}^{c}(M)$, by $G_{1}^{*}$ we denote the subgroup of $G_{1}$ consisting of $h \in G$ which admits a path $h_{t} \in G(t \in[0,1])$ such that $h_{0}=i d_{M}, h_{1}=h$ and there exists $K \in \mathcal{K}(M)$ with $\operatorname{supp} h_{t} \subset K(t \in[0,1])$. Since $G$ is a topological group, for any continuous maps $\varphi, \psi: P \rightarrow G$, the composition $\psi \varphi: P \rightarrow G,(\psi \varphi)_{p}=\psi_{p} \varphi_{p}$ and the inverse $\varphi^{-1}: P \rightarrow G,\left(\varphi^{-1}\right)_{p}=\varphi_{p}^{-1}$ are also continuous maps. We say that a map $\varphi: P \rightarrow G$ (or a map $\mu: P \rightarrow \Omega^{n}(M)$ ) has locally common compact support in $V \subset M$ if for each point $p \in P$ there exists a neighborhood $U$ of $p$ in $P$ and $K \in \mathcal{K}(V)$ such that $\operatorname{supp} \varphi_{q} \subset K\left(\right.$ or $\left.\operatorname{supp} \mu_{q} \subset K\right)(q \in U)$.

2.2. Ends of manifolds (cf. [4]). Suppose $M$ is a noncompact, connected $C^{\infty}$ $n$-manifold, possibly with corners. An end of $M$ is a function $e$ which assigns an $e(K) \in \mathcal{C}(M-K)$ to each $K \in \mathcal{K}(M)$ such that $e\left(K_{1}\right) \supset e\left(K_{2}\right)$ if $K_{1} \subset K_{2}$. The set of ends of $M$ is denoted by $E_{M}$. The end compactification of $M$ is the space $\bar{M}=M \cup E_{M}$ endowed with the topology defined by the following conditions: (a) $M$ is an open subspace of $\bar{M}$, (b) the fundamental open neighborhoods of $e \in E_{M}$ are given by

$$
N(e, K)=e(K) \cup\left\{e^{\prime} \in E_{M} \mid e^{\prime}(K)=e(K)\right\} \quad(K \in \mathcal{K}(M)) .
$$

Then, (i) $\bar{M}$ is a connected, locally connected, compact, metrizable space, (ii) $M$ is a dense open subset of $\bar{M}$ and (iii) $E_{M}$ is a compact 0-dimensional subset of $\bar{M}$. Thus, for any metric $d$ on $\bar{M}$ and any $\varepsilon>0$ there exists a neighborhood $U$ of $E_{M}$ in $\bar{M}$ such that $\operatorname{diam}_{d} C<\varepsilon(C \in \mathcal{C}(U))$.

Let $\operatorname{Map}(\bar{M})$ denote the space of continuous maps $f: \bar{M} \rightarrow \bar{M}$ and let $\mathcal{H}(\bar{M})$ denote the group of homeomorphisms $h$ of $\bar{M}$ onto itself. These spaces are endowed with the compact-open topology. Any metric $d$ on $\bar{M}$ induces the sup-metric $d$ on $\operatorname{Map}(\bar{M})$, which is compatible with the compact-open topology. For $A \subset \bar{M}$ we set $\operatorname{Map}_{A}(\bar{M})=\left\{f \in \operatorname{Map}(\bar{M})|f|_{A}=i d_{A}\right\}$ and $\mathcal{H}_{A}(\bar{M})=\left\{h \in \mathcal{H}(\bar{M})|h|_{A}=i d_{A}\right\}$.

For $h \in \mathcal{D}(M)$ and $e \in E_{M}$ we define $h(e) \in E_{M}$ by

$$
h(e)(K)=h\left(e\left(h^{-1}(K)\right)\right) \in \mathcal{C}(M-K) \quad(K \in \mathcal{K}(M)) .
$$

Then $h$ has a unique extension $\bar{h} \in \mathcal{H}(\bar{M})$ defined by $\bar{h}(e)=h(e)\left(e \in E_{M}\right)$ and the map $\mathcal{D}(M) \rightarrow \mathcal{H}(\bar{M}): h \mapsto \bar{h}$ is a continuous group homomorphism. If $h \in \mathcal{D}(M)_{1}$, then $\bar{h} \in \mathcal{H}_{E_{M}}(\bar{M})$. For $A, B \subset \bar{M}$ we set

$$
\mathcal{D}_{A}(M, B)=\left\{h \in \mathcal{D}(M)|\bar{h}|_{A}=i d_{A}, \bar{h}(B)=B\right\} .
$$

For each $C \in \mathcal{B}_{c}(M)$ we set

$$
\bar{C}=C \cup E_{C}, \quad E_{C}=\left\{e \in E_{M} \mid e(K) \subset C \text { for some } K \in \mathcal{K}(M)\right\} .
$$

Then, $E_{C}$ is open and closed in $E_{M}$ and $\bar{C}$ is a neighborhood of $E_{C}$ in $\bar{M}$. 
For $A, B \in \mathcal{B}(M)$ we write $A \sim_{c} B$ if the symmetric difference $A \Delta B=(A-$ $B) \cup(B-A)$ is relatively compact (i.e., has the compact closure) in $M$. This is an equivalence relation, and for $C, D \in \mathcal{B}_{c}(M)$ we have (i) $C \sim_{c} D$ iff $E_{C}=E_{D}$ and (ii) $C \sim_{c} h(C)$ for any $h \in \mathcal{D}_{E_{M}}(M)$.

For $\omega \in \mathcal{M}_{g}^{\partial}(M)$ (or $\omega \in \mathcal{V}^{+}(M)$ when $M$ is oriented), we say that an end $e \in E_{M}$ is $\omega$-finite if $\omega(e(K))<\infty$ for some $K \in \mathcal{K}(M)$. We set $E_{M}^{\omega}=\left\{e \in E_{M} \mid\right.$ $e: \omega$-finite $\}$. Then $E_{M}^{\omega}$ is an open subset of $E_{M}$, and for $C \in \mathcal{B}_{c}(M)$ we have that $\omega(C)<\infty$ iff $E_{C} \subset E_{M}^{\omega}$.

2.3. Volume transfer and end charge homomorphism. First we introduce a quantity which measures volume transfer by diffeomorphisms (cf. 12, Section 3.2]). Suppose $M$ is a connected oriented $C^{\infty} n$-manifold possibly with corners and $\omega \in \mathcal{V}^{+}(M)$. For $A, B \in \mathcal{B}(M)$ we write $A \sim_{\omega} B$ if $\omega(A \Delta B)<\infty$. This is an equivalence relation, and $A \sim_{c} B$ implies $A \sim_{\omega} B$. If $A \sim_{\omega} B$, then we can define the following quantity:

$$
J^{\omega}(A, B)=\omega(A-B)-\omega(B-A) \in \mathbb{R} .
$$

If $C \in \mathcal{B}_{c}(M)$ and $h \in \mathcal{D}_{E_{M}}(M)$, then $J^{\omega}\left(h^{-1}(C), C\right)$ is just the total $\omega$-mass transferred into $C$ by $h$. If $A \in \mathcal{B}(M)$ and $f, g \in \mathcal{D}^{c}(M)$, then $J^{\omega}\left(f^{-1}(A), g^{-1}(A)\right)=$ $\left(f_{*} \omega-g_{*} \omega\right)(A)$.

The quantity $J^{\omega}$ has the following formal properties:

Lemma 2.1. Suppose $A, B, C, D \in \mathcal{B}(M)$.

(1) If $A \sim_{\omega} B$, then

(i) $\omega(A)=J^{\omega}(A, B)+\omega(B)$.

(ii) If $\omega(A)<\infty$, then $\omega(B)<\infty$ and $J^{\omega}(A, B)=\omega(A)-\omega(B)$.

(2) If $A \sim_{\omega} B \sim_{\omega} C$, then $J^{\omega}(A, B)+J^{\omega}(B, C)=J^{\omega}(A, C)$.

(3) If $A \sim_{\omega} C, B \sim_{\omega} D$, then

(i) $A \cup B \sim \sim_{\omega} C \cup D$.

(ii) If $A \cap B=C \cap D=\emptyset$, then $J^{\omega}(A \cup B, C \cup D)=J^{\omega}(A, C)+J^{\omega}(B, D)$.

(4) If $h \in \mathcal{D}(M)$ and $A \sim_{h_{*} \omega} B$, then $h^{-1}(A) \sim_{\omega} h^{-1}(B)$ and $J^{h_{*} \omega}(A, B)=$ $J^{\omega}\left(h^{-1}(A), h^{-1}(B)\right)$.

Lemma 2.2. Suppose $A, B \in \mathcal{B}_{c, 0}(M)$ and $A \sim_{c} B$. Then the following function is continuous:

$$
\Phi: \mathcal{V}^{+}(M) \times \mathcal{D}_{E_{M}}(M)^{2} \longrightarrow \mathbb{R}: \quad \Phi(\mu, f, g)=J^{\mu}(f(A), g(B)) .
$$

Lemma 2.1] is a special case of [12, Lemma 3.1], while Lemma 2.2 follows directly from [12, Lemma 3.2] since the canonical maps $\mathcal{V}^{+}(M) \rightarrow \mathcal{M}_{g}^{\partial}(M, \omega$-reg) and $\mathcal{D}_{E_{M}}(M) \rightarrow \mathcal{H}_{E_{M}}(M, \omega$-reg) are continuous.

Next we recall basic properties of the end charge homomorphism defined in [1. Section 14]. Suppose $M$ is a noncompact connected oriented $C^{\infty} n$-manifold, possibly with corners, and $\omega \in \mathcal{V}^{+}(M)$. The symbol $\mathcal{Q}\left(E_{M}\right)$ denotes the algebra of clopen subsets of $E_{M}$. An end charge of $M$ is a finitely additive signed measure $c$ on $\mathcal{Q}\left(E_{M}\right)$. That is, it is a function $c: \mathcal{Q}\left(E_{M}\right) \rightarrow \mathbb{R}$ which satisfies the following condition:

$$
c(F \cup G)=c(F)+c(G) \text { for } F, G \in \mathcal{Q}\left(E_{M}\right) \text { with } F \cap G=\emptyset .
$$

Let $\mathcal{S}(M)$ denote the space of end charges $c$ of $M$ with the weak topology (or the product topology). This topology is the weakest topology such that the function 
$\mathcal{S}(M) \rightarrow \mathbb{R}: c \mapsto c(F)$ is continuous for any $F \in \mathcal{Q}\left(E_{M}\right)$. We set

$$
\mathcal{S}(M ; \omega)=\left\{c \in \mathcal{S}(M) \mid \text { (i) } c\left(E_{M}\right)=0 \text {, (ii) } c(F)=0\left(F \in \mathcal{Q}\left(E_{M}\right), F \subset E_{M}^{\omega}\right)\right\} \text {. }
$$

Then $\mathcal{S}(M)$ is a topological linear space and $\mathcal{S}(M ; \omega)$ is a linear subspace of $\mathcal{S}(M)$.

For $h \in \mathcal{D}_{E_{M}}(M ; \omega)$ the end charge $c_{h}^{\omega} \in \mathcal{S}(M ; \omega)$ is defined as follows: For any $F \in \mathcal{Q}\left(E_{M}\right)$ there exists $C \in \mathcal{B}_{c}(M)$ with $E_{C}=F$. Since $C \sim_{c} h(C)$, we can define as

$$
c_{h}^{\omega}(F)=J^{\omega}\left(h^{-1}(C), C\right)\left(=J^{\omega}(C, h(C))=\omega(C-h(C))-\omega(h(C)-C)\right) \in \mathbb{R} .
$$

This quantity is independent of the choice of $C$.

Proposition 2.1. The map $c^{\omega}: \mathcal{D}_{E_{M}}(M ; \omega) \longrightarrow \mathcal{S}(M ; \omega)$ is a continuous group homomorphism ([1, Section 14.9, Lemma 14.21 (iv)]).

2.4. Action of diffeomorphism groups on spaces of volume forms. Suppose $M$ is an oriented $C^{\infty} n$-manifold, possibly with corners. The group $\mathcal{D}^{+}(M)$ acts continuously on $\mathcal{V}^{+}(M)$ by $h \cdot \mu=h_{*} \mu$. The subgroup $\mathcal{D}(M ; \omega)$ coincides with the stabilizer of $\omega \in \mathcal{V}^{+}(M)$. For $m \in(0, \infty]$ we obtain the invariant subspace $\mathcal{V}^{+}(M ; m)=\left\{\mu \in \mathcal{V}^{+}(M) \mid \mu(M)=m\right\}$. For $\omega \in \mathcal{V}^{+}(M ; m)$ we have the orbit map $\pi_{\omega}: \mathcal{D}^{+}(M) \rightarrow \mathcal{V}^{+}(M ; m), \pi_{\omega}(h)=h_{*} \omega$. Moser's theorem asserts that this orbit map has a continuous section in case $M$ is a compact connected oriented $C^{\infty} n$-manifold (J. Moser [9], A. Banyaga 2, 3]). In this article we apply the next version. The following notation are used for a collar $E=S \times[a, b]$ and $A \subset S$ :

$$
\begin{gathered}
E_{A}=\{(x, t) \in E \mid x \in A\}, \quad E_{A}^{+}=\left\{(x, t) \in E_{A} \mid t \geq 0\right\}, \\
E_{A}^{-}=\left\{(x, t) \in E_{A} \mid t \leq 0\right\} \text { and } \\
E_{A}[\delta, \varepsilon]=\left\{(x, t) \in E_{A} \mid t \in[\delta(x), \varepsilon(x)]\right\} \quad \text { for functions } \delta, \varepsilon: S \rightarrow[a, b] .
\end{gathered}
$$

Theorem 2.1. Suppose $M$ is a compact connected oriented $C^{\infty} n$-manifold, possibly with corners, and $E=\partial M \times[0,1]$ is a collar of $\partial M$. Suppose $\mu, \nu: P \rightarrow \mathcal{V}^{+}(M)$ are continuous maps and $\varepsilon: P \rightarrow(0,1 / 2)$ is a continuous function such that for each $p \in P$,

$$
\text { (i) } \mu_{p}(M)=\nu_{p}(M) \text { and (ii) } \mu_{p}=\nu_{p} \text { on } E\left[0,2 \varepsilon_{p}\right] \text {. }
$$

Then there exists a continuous map $\varphi: P \rightarrow \mathcal{D}_{\partial}(M)_{1}$ such that for each $p \in P$
(1) $\varphi_{p_{*}} \mu_{p}=\nu_{p}$,
(2) $\varphi_{p}=i d_{M}$ on $E\left[0, \varepsilon_{p}\right]$,
(3) if $\mu_{p}=\nu_{p}$, then $\varphi_{p}=i d_{M}$.

Theorem 2.1 is complemented by the next lemma, which is a parametrized version of [10, Lemma A2].

Lemma 2.3. Let $M$ be an oriented $C^{\infty} n$-manifold possibly with boundary.

(1) Suppose $S$ is a proper $(n-1)$-submanifold of $M, K$ is a closed subset of $S$, $U$ is a neighborhood of $K$ in $S$ and $E=S \times[-1,1]$ is a bicollar neighborhood of $S$ in $M$. Then, for any continuous maps $\mu, \nu: P \rightarrow \mathcal{V}^{+}(M)$ there exist continuous maps $\varphi: P \rightarrow \mathcal{D}_{S \cup\left(M-E_{U}\right)}(M)_{1}$ and $\varepsilon: P \rightarrow C^{\infty}(S,(0,1))$ such that for each $p \in P$,

(i) $\varphi_{p_{*}} \mu_{p}=\nu_{p}$ on $E_{K}\left[-\varepsilon_{p}, \varepsilon_{p}\right]$,

(ii) for each $x \in S$, (a) $\varphi_{p}\left(E_{x}^{ \pm}\right)=E_{x}^{ \pm}$and (b) if $\mu_{p}=\nu_{p}$ on $E_{x}^{ \pm}$, then $\varphi_{p}=i d$ on $E_{x}^{ \pm}$; in particular, if $\mu_{p}=\nu_{p}$ on $E^{ \pm}$, then $\varphi_{p}=$ id on $E^{ \pm}$. 
(2) Suppose $K$ is a closed subset of $\partial M, U$ is a neighborhood of $K$ in $\partial M$ and $E=\partial M \times[0,1]$ is a collar of $\partial M$ in $M$. Then, for any continuous maps $\mu, \nu: P \rightarrow \mathcal{V}^{+}(M)$ there exist continuous maps $\psi: P \rightarrow \mathcal{D}_{\partial M \cup\left(M-E_{U}\right)}(M)_{1}$ and $\varepsilon: P \rightarrow C^{\infty}(\partial M,(0,1))$ such that for each $p \in P$,

(i) $\psi_{p_{*}} \mu_{p}=\nu_{p}$ on $E_{K}\left[0, \varepsilon_{p}\right]$,

(ii) for each $x \in \partial M$, (a) $\psi_{p}\left(E_{x}\right)=E_{x}$ and (b) if $\mu_{p}=\nu_{p}$ on $E_{x}$, then $\psi_{p}=i d$ on $E_{x}$; in particular, if $\mu_{p}=\nu_{p}$ on $E$, then $\psi_{p}=i d_{M}$.

Remark 2.1. In Lemma 2.3 (1), the map $\varphi_{p}$ does not fix $\partial M$ pointwise. To remedy this point, we can apply (2) before (1) to obtain the following conclusion:

(3) Suppose $S$ is a proper $(n-1)$-submanifold of $M$ and $E=S \times[-1,1]$ is a bicollar neighborhood of $S$ in $M$. Then, for any continuous maps $\mu, \nu$ : $P \rightarrow \mathcal{V}^{+}(M)$ there exist continuous maps $\varphi^{\prime}: P \rightarrow \mathcal{D}_{\partial M \cup(M-E)}(M, S)_{1}$ and $\varepsilon^{\prime}: P \rightarrow C^{\infty}(S,(0,1))$ such that for each $p \in P$,

(i) $\varphi_{p_{*}}^{\prime} \mu_{p}=\nu_{p}$ on $E\left[-\varepsilon_{p}^{\prime}, \varepsilon_{p}^{\prime}\right]$,

(ii) if $\mu_{p}=\nu_{p}$, then $\varphi_{p}^{\prime}=i d_{M}$.

For completeness we include the proofs of Theorem 2.1 and Lemma 2.3 in the Appendix.

To deal with the noncompact case, it is necessary to include the information on the ends of manifolds $(4,8])$. Suppose $M$ is a noncompact, connected oriented $C^{\infty}$ $n$-manifold, possibly with corners. For $m \in(0, \infty]$ and an open subset $F$ of $E_{M}$, we set

$\mathcal{V}^{+}(M ; F)=\left\{\mu \in \mathcal{V}^{+}(M) \mid E_{M}^{\mu}=F\right\} \quad$ and $\quad \mathcal{V}^{+}(M ; m, F)=\mathcal{V}^{+}(M ; m) \cap \mathcal{V}^{+}(M ; F)$.

To distinguish topologies, by $\mathcal{V}^{+}(M ; F)_{w}$ we denote the space $\mathcal{V}^{+}(M ; F)$ endowed with the compact-open $C^{\infty}$-topology. If $C \in \mathcal{B}_{c}(M)$ and $E_{C} \subset F$, then we obtain the function

$$
\Phi_{C}: \mathcal{V}^{+}(M ; F)_{w} \rightarrow \mathbb{R}, \quad \Phi_{C}(\mu)=\mu(C) .
$$

It is easily seen that this map is not continuous if $C$ is not compact. In [4] R. Belranga overcame this problem by introducing a stronger topology called the finite-ends weak topology (on the spaces of Radon measures). Consider the subspace $M \cup F \subset \bar{M}$.

The finite-ends weak topology on the space $\mathcal{M}_{g}^{\partial}(M ; F)=\left\{\mu \in \mathcal{M}_{g}^{\partial}(M) \mid E_{M}^{\mu}=\right.$ $F\}$ is the weakest topology such that

(0) the function $\Phi_{f}: \mathcal{M}_{g}^{\partial}(M ; F) \rightarrow \mathbb{R}: \Phi_{f}(\mu)=\int_{M} f d \mu$ is continuous for any continuous function $f: M \cup F \rightarrow \mathbb{R}$ with compact support.

Let $\mathcal{M}_{g}^{\partial}(M ; F)_{\text {ew }}$ denote the space $\mathcal{M}_{g}^{\partial}(M ; F)$ endowed with the finite-ends weak topology.

Definition 2.1. The finite-ends weak $C^{\infty}$ topology on $\mathcal{V}^{+}(M ; F)$ is the weakest topology such that

(i) the identity map $i d: \mathcal{V}^{+}(M ; F) \rightarrow \mathcal{V}^{+}(M ; F)_{w}$ is continuous,

(ii) the function $\Phi_{f}: \mathcal{V}^{+}(M ; F) \rightarrow \mathbb{R}: \Phi_{f}(\mu)=\int_{M} f \mu$ is continuous for any continuous function $f: M \cup F \rightarrow \mathbb{R}$ with compact support. 
Let $\mathcal{V}^{+}(M ; F)_{\text {ew }}$ denote the space $\mathcal{V}^{+}(M ; F)$ endowed with the finite-ends weak $C^{\infty}$ topology. From the definition it follows that the canonical map

$$
\mathcal{V}^{+}(M ; F)_{e w} \rightarrow \mathcal{M}_{g}^{\partial}(M ; F)_{e w}
$$

is continuous.

Lemma 2.4. If $C \in \mathcal{B}_{c}(M)$ and $E_{C} \subset F$, then the function $\Phi_{C}: \mathcal{V}^{+}(M ; F)_{e w} \rightarrow$ $\mathbb{R}, \Phi_{C}(\mu)=\mu(C)$ is continuous.

Proof. Let $K=\operatorname{cl}_{M} C \cup E_{C}$ and take an open neighborhood $U$ of $\operatorname{Fr}_{M} C$ in $M$ such that $\operatorname{cl}_{M} U$ is compact. Then $K, K-U \in \mathcal{K}(M \cup F)$ and $\bar{C}=C \cup E_{C}$ is a neighborhood of $K-U$ in $M \cup F$. Thus, there exists a continuous function $f: M \cup F \rightarrow[0,1]$ such that $f=1$ on $K-U$ and $f=0$ on $(M \cup F) \backslash \bar{C}$.

Since $f$ has compact support in $K$, by condition (ii) in Definition 2.1 we have the continuous function $\Phi_{f}: \mathcal{V}^{+}(M ; F)_{e w} \rightarrow \mathbb{R}$. Since the Borel measurable function $g=\chi_{C}-f: M \rightarrow[0,1]$ has compact support in $c l_{M} U$, by condition (i) in Definition 2.1 the map $\Phi_{g}: \mathcal{V}^{+}(M ; F)_{e w} \rightarrow \mathbb{R}$ is continuous. Thus $\Phi_{C}=\Phi_{\chi_{C}}=\Phi_{f}+\Phi_{g}$ is also continuous.

The group $\mathcal{D}^{+}(M ; F)$ acts continuously on $\mathcal{V}^{+}(M ; F)_{e w}$ by $h \cdot \mu=h_{*} \mu$. The subspace $\mathcal{V}^{+}(M ; m, F)_{e w}$ is invariant under this action, and for $\omega \in \mathcal{V}^{+}(M ; m, F)_{\text {ew }}$ we have the orbit map

$$
\pi_{\omega}: \mathcal{D}^{+}(M ; F) \rightarrow \mathcal{V}^{+}(M ; m, F)_{e w}, \pi_{\omega}(h)=h_{*} \omega .
$$

The subgroup $\mathcal{D}^{+}(M ; \omega)$ coincides with the stabilizer of $\omega$. The transitivity of this action on $\mathcal{V}^{+}(M ; m, F)_{\text {ew }}$ is proved in 8 .

For the sake of notational simplicity we follow the next convention in the subsequent sections. For continuous maps $\varphi: P \rightarrow \mathcal{D}^{+}(M), \mu, \nu: P \rightarrow \mathcal{V}^{+}(M)$ and an $n$-submanifold $N$ of $M$, we obtain continuous maps $\varphi_{*} \mu: P \rightarrow \mathcal{V}^{+}(M)$, $\left(\varphi_{*} \mu\right)_{p}=\varphi_{p_{*}} \mu_{p}$ and $\left.\nu\right|_{N}: P \rightarrow \mathcal{V}^{+}(N),\left(\left.\nu\right|_{N}\right)_{p}=\left.\nu_{p}\right|_{N}$.

Notation 2.1. The identities for maps $\left(\varphi_{*} \mu\right)(N)=\nu(N),\left.\varphi\right|_{N}=i d_{N}$, etc. mean that $\left(\varphi_{p_{*}} \mu_{p}\right)(N)=\nu_{p}(N)(p \in P),\left.\varphi_{p}\right|_{N}=i d_{N}(p \in P)$, etc.

\section{REALIZATION THEOREM FOR VOLUME TRANSFER TOWARD ENDS}

In this section we discuss the realization problem of volume transfer toward ends by diffeomorphisms. The main theorem, Theorem 3.1, is applied in the next section to deduce Theorems 1.1 and 1.2

3.1. Volume transfer by engulfing isotopy. Suppose $M$ is a connected oriented $C^{\infty} n$-manifold, possibly with corners, and $d$ is any metric on $\bar{M}$. Consider a decomposition $M=L \cup_{S} N$ such that

(i) $L$ and $N$ are connected $C^{\infty} n$-submanifolds of $M$,

(ii) $S \equiv L \cap N=\operatorname{Fr}_{M} L=\operatorname{Fr}_{M} N$, which is a compact proper $C^{\infty}(n-1)$ submanifold of $M$.

Lemma 3.1. There exists a continuous map $f:(-\infty, \infty) \rightarrow \mathcal{D}_{\partial}^{c}(M)_{1}^{*}$ such that

(1) $f_{0}=i d, \quad f_{s}(L) \varsubsetneqq f_{t}(L)(s<t)$,

(2) there exists a subpolyhedron $F$ of $M$ (with respect to a $C^{\infty}$-triangulation of $M)$ such that

(i) $\operatorname{dim} F=n-1$ and $\partial M \subset F$, 
(ii) the map $f$ has a locally common compact support in $M-F$ (i.e., for any $T>0$ there exists $K \in \mathcal{K}(M-F)$ such that $\operatorname{supp} f_{t} \subset K$ $(t \in[-T, T]))$,

(iii) for any $K \in \mathcal{K}(M-F)$ there exist $-\infty<s<t<\infty$ with $K \subset$ $f_{t}(L)-f_{s}(L)$

(3) $\left\{f_{t}\right\}_{-\infty<t<\infty}$ is equi-continuous with respect to $\left.d\right|_{M}$.

Proof. Take a $C^{\infty}$-triangulation $\tau$ of $M$ such that $L$ and $N$ are subcomplexes of $\tau$. For $k=0,1, \cdots, n$ let $\tau(k)$ denote the set of $k$-simplices of $\tau$, and for $t \in \tau$ let $\tau_{t}=\{s \in \tau \mid t \varsubsetneqq s\}$. Consider the barycentric subdivision $\tau^{\prime}$ of $\tau$. The barycenter of a simplex $s \in \tau$ is denoted by $b(s)$. If $s_{1}, s_{2} \in \tau$ and $s_{1} \varsubsetneqq s_{2}$, then we have the associated 1-simplex $\left\langle b\left(s_{1}\right), b\left(s_{2}\right)\right\rangle$ of $\tau^{\prime}$. The dual 0-cell $e(s)$ associated with an $s \in \tau(n)$ is the barycenter $b(s)$ of $s$. The dual 1-cell $e(t)$ associated with a $t \in \tau(n-1)$ is the union of 1-simplices $\langle b(t), b(s)\rangle\left(s \in \tau_{t}\right)$ of $\tau^{\prime}$. Note that $\# \tau_{t}=1$ if $t \subset \partial M$ and $\# \tau_{t}=2$ otherwise. The dual 1-skeleton $S_{M}$ of $\tau$ is the union of all dual 0-cells and dual 1-cells of $\tau$. A maximal tree of $S_{M}$ is a tree $T$ of the graph $S_{M}$ which is a union of dual 1-cells of $\tau$ and includes all dual 0-cells of $\tau$.

Consider the triangulation $\left.\tau\right|_{L}$ of $L$ and $\left.\tau\right|_{N}$ of $N$. Let $S_{L}$ and $S_{N}$ be the dual 1-skeletons of $\left.\tau\right|_{L}$ and $\left.\tau\right|_{N}$ and take maximal trees $T_{L}$ of $S_{L}$ and $T_{N}$ of $S_{N}$ such that $T_{L} \subset \operatorname{Int} L$ and $T_{N} \subset \operatorname{Int} N$. Take any $(n-1)$-simplex $\left.s_{0} \in \tau\right|_{S}$, and let $T_{S}$ denote the dual 1-cell associated to $s_{0}$. Then $T=T_{L} \cup T_{S} \cup T_{N}$ is a maximal tree of the dual 1-skeleton $S_{M}$ of $\tau$. Let $F=\cup\{s \in \tau \mid s \cap T=\emptyset\}$ and $U=M-F$. Then $F$ is a subpolyhedron of $M$ such that $\operatorname{dim} F=n-1, \partial M \subset F, S \cap F=S-$ Int $s_{0}$, and $U$ is an open regular neighborhood of $T$, which is an open $C^{\infty} n$-cell.

Consider the decomposition of the end compactification $\bar{M}=\bar{L} \cup_{S} \bar{N}$. Let $\bar{F}=F \cup E_{M}, \bar{F}_{L}=\bar{F} \cap \bar{L}$ and $\bar{F}_{N}=\bar{F} \cap \bar{N}$. Using $(U, T)$, we can find an engulfing pesudo-isotopy $\bar{f}:[-\infty, \infty] \rightarrow \operatorname{Map}_{\bar{F}}(\bar{M})$ in $\bar{M}$ such that

(i) (a) $\bar{f}_{0}=i d$ and $\bar{f}_{t} \in \mathcal{H}_{\bar{F}}(\bar{M})(t \in(-\infty, \infty))$,

(b) for any $T \in(0, \infty)$ there exists $K \in \mathcal{K}(U)$ such that $\operatorname{supp} \bar{f}_{t} \subset K$ $(t \in[-T, T])$,

(ii) (a) $f_{t}=\left.\bar{f}_{t}\right|_{M} \in \mathcal{D}_{F}^{c}(M)(t \in(-\infty, \infty))$,

(b) the map $f:(-\infty, \infty) \rightarrow \mathcal{D}_{F}^{c}(M)_{1}^{*}: t \mapsto f_{t}$ is continuous,

(c) $f$ has locally common compact support in $U$,

(iii) (a) $\bar{f}_{s}(\bar{L}) \varsubsetneqq \bar{f}_{t}(\bar{L})$ for $-\infty \leq s<t \leq \infty$,

(b) for any $K \in \mathcal{K}(U)$ there exist $-\infty<s<t<\infty$ such that $K \subset$ $\bar{f}_{t}(\bar{L})-\bar{f}_{s}(\bar{L})$

(c) $\bar{f}_{-\infty}(\bar{L})=\bar{F}_{L}, \bar{f}_{-\infty}(\bar{N})=\bar{M}$, and $\bar{f}_{\infty}(\bar{L})=\bar{M}, \bar{f}_{\infty}(\bar{N})=\bar{F}_{N}$.

Since the compact family $\left\{\bar{f}_{t}\right\}_{-\infty \leq t \leq \infty}$ is equi-continuous with respect to $d$, the family $\left\{f_{t}\right\}_{-\infty<t<\infty}$ is also equi-continuous with respect to $\left.d\right|_{M}$. Hence, the map $f$ given in (ii) satisfies the required conditions.

Now we use the isotopy $h_{t}=f_{t}^{-1}$ to transfer volume on $M$. Consider the continuous maps

(1) $\lambda: \mathcal{V}^{+}(M) \times \mathbb{R} \rightarrow \mathbb{R}, \quad \lambda(\mu, t)=J^{\mu}\left(h_{t}^{-1}(L), L\right)=\left\{\begin{array}{rr}\mu\left(f_{t}(L)-L\right) & (t \geq 0) \\ -\mu\left(L-f_{t}(L)\right) & (t \leq 0)\end{array}\right.$,

$(2) \mathcal{W}^{+}(M)=\left\{(\mu, a) \in \mathcal{V}^{+}(M) \times \mathbb{R} \mid a \in(-\mu(L), \mu(N))\right\}$ (an open subset of $\left.\mathcal{V}^{+}(M) \times \mathbb{R}\right)$, 
(3) $t: \mathcal{W}^{+}(M) \rightarrow \mathbb{R}, \quad t(\mu, a)=\lambda(\mu, *)^{-1}(a) \quad$ (i.e., $t=t(\mu, a)$ iff $a=\lambda(\mu, t)$ ),

(4) $H: \mathcal{W}^{+}(M) \rightarrow \mathcal{D}_{\partial}^{c}(M)_{1}^{*}, \quad H_{(\mu, a)}=h_{t(\mu, a)}$.

Since the map $\lambda(\mu, *): \mathbb{R} \rightarrow(-\mu(L), \mu(N))$ is a monotonically increasing homeomorphism, the map $t$ is well defined. Note that $h_{0}=i d_{M}$ and $t(\mu, 0)=0$.

Lemma 3.2. The map $H$ has the following properties:

(i) $H$ has locally common compact support in $\operatorname{Int} M$.

(ii) $\left\{H_{(\mu, a)}{ }^{-1}\right\}_{(\mu, a)}$ is equi-continuous with respect to $\left.d\right|_{M}$.

(iii) $J^{\mu}\left(H_{(\mu, a)}^{-1}(L), L\right)=a$.

(iv) $H_{(\mu, a)}=i d_{M}$ iff $a=0$.

Remark 3.1. The continuous map

$$
\widetilde{H}: \mathcal{W}^{+}(M) \times[0,1] \rightarrow \mathcal{D}_{\partial}^{c}(M)_{1}^{*}, \quad \widetilde{H}_{(\mu, a), s}=H_{(\mu, s a)}
$$

provides a homotopy from $\widetilde{H}_{0} \equiv i d_{M}$ to $\widetilde{H}_{1}=H$ with locally common compact support in Int $M$.

3.2. Fundamental deformation lemmas. Suppose $M$ is a connected oriented $C^{\infty} n$-manifold, possibly with corners, $d$ is any metric on $\bar{M}$ and $N$ is a connected $C^{\infty} n$-submanifold of $M$ such that $\operatorname{Fr}_{M} N$ is compact. Let $\mathcal{C}\left(N^{c}\right)=\left\{A_{1}, \cdots, A_{m}\right\}$.

Lemma 3.3. Suppose $\mu: P \rightarrow \mathcal{V}^{+}(M)$ is a continuous map and $a(i): P \rightarrow \mathbb{R}$ $(i=0,1, \cdots, m)$ are continuous functions such that

(a) $\sum_{i=0}^{m} a(i)=0$,

(b) $a(0)>-\mu(N), a(i)>-\mu\left(A_{i}\right)(i=1, \cdots, m)$.

Then there exists a continuous map $\varphi: P \rightarrow \mathcal{D}_{\partial}^{c}(M)_{1}^{*}$ such that

(1) (i) $\varphi$ has locally common compact support in Int $M$ (and there exists a homotopy from $\varphi$ to the constant map $i d_{M}$ with locally common compact support in $\operatorname{Int} M)$,

(ii) the family $\left\{\varphi_{p}^{-1}\right\}_{p}$ is equi-continuous with respect to $\left.d\right|_{M}$,

(2) (i) $J^{\mu}\left(\varphi^{-1}(N), N\right)=a(0)$,

(ii) $J^{\mu}\left(\varphi^{-1}\left(A_{i}\right), A_{i}\right)=a(i)(i=1, \cdots, m)$,

(3) if $p \in P$ and $a_{p}(i)=0(i \in\{1, \cdots, m\})$, then $\varphi_{p}=i d_{M}$.

Remark 3.2. In Lemma 3.3 we note the following points:

(A) Condition (2)(ii) implies (2)(i). This follows from condition (a) and the equation

$J^{\mu}\left(\varphi^{-1}(N), N\right)+\sum_{i} J^{\mu}\left(\varphi^{-1}\left(A_{i}\right), A_{i}\right)=J^{\mu}\left(\varphi^{-1}(M), M\right)=J^{\mu}(M, M)=0$.

(B) If condition (b) is replaced by

(b) $a(0)<\mu(N), a(i)<\mu\left(A_{i}\right)(i=1, \cdots, m)$, then condition (2) is replaced by

$(2)^{\prime} \quad$ (i) $J^{\mu}\left(N, \varphi^{-1}(N)\right)=a(0)$,

(ii) $J^{\mu}\left(A_{i}, \varphi^{-1}\left(A_{i}\right)\right)=a(i)(i=1, \cdots, m)$.

This is verified by applying Lemma 3.3 to the functions $-a_{i}$. 
Proof of Lemma 3.3. [1] First we verify the case $m=1$. The argument in Section 3.1 can be applied to $M=A_{1} \cup N$ so as to yield the associated map $H: \mathcal{W}^{+}(M) \rightarrow$ $\mathcal{D}_{\partial}^{c}(M)_{1}^{*}$. From conditions (a) and (b) it follows that $\left(\mu_{p}, a_{p}(1)\right) \in \mathcal{W}^{+}(M)$ (i.e., $\left.a_{p}(1) \in\left(-\mu_{p}\left(A_{1}\right), \mu_{p}(N)\right)\right)$ for each $p \in P$. Thus, we obtain the composition

$$
\varphi: P \rightarrow \mathcal{D}_{\partial}^{c}(M)_{1}^{*}, \quad \varphi_{p}=H_{\left(\mu_{p}, a_{p}(1)\right)}(p \in P) .
$$

The required properties of $\varphi$ are verified by Lemma 3.2 and Remarks 3.1 and 3.2(A).

[2] We proceed by the induction on $m$. For $m=0$ we can take $\varphi \equiv i d_{M}$. Suppose $m \geq 1$ and assume that the assertion holds for $m-1$. To prove the assertion for $m$, first we apply the inductive hypothesis to the data : $M=\left(N \cup A_{1}\right) \cup A_{2} \cup \cdots \cup A_{m}$ and $a(0)+a(1), a(2), \cdots, a(m)$ to yield a map $\psi: P \rightarrow \mathcal{D}_{\partial}^{c}(M)_{1}^{*}$ which satisfies conditions (1), (3) (for $a(0)+a(1), a(2), \cdots, a(m)$ ) and

(2) $)_{1} \quad$ (i) $J^{\mu}\left(\psi^{-1}\left(N \cup A_{1}\right), N \cup A_{1}\right)=a(0)+a(1)$,

$$
\text { (ii) } J^{\mu}\left(\psi^{-1}\left(A_{i}\right), A_{i}\right)=a(i)(i=2, \cdots, m) \text {. }
$$

Next we apply [1] (the case $m=1$ ) to the data : $M_{1}=N \cup A_{1},\left.\quad\left(\psi_{*} \mu\right)\right|_{M_{1}}: P \rightarrow$ $\mathcal{V}^{+}\left(M_{1}\right)$ and the functions $b(0), b(1): P \rightarrow \mathbb{R}$ defined by

$$
b(0)=a(0)-J^{\mu}\left(\psi^{-1}(N), N\right), \quad b(1)=a(1)-J^{\mu}\left(\psi^{-1}\left(A_{1}\right), A_{1}\right) .
$$

Note that

(a) $b(0)+b(1)=a(0)+a(1)-J^{\mu}\left(\psi^{-1}\left(N \cup A_{1}\right), N \cup A_{1}\right)=0$,

(b) $b(0)=a(0)-J^{\mu}\left(\psi^{-1}(N), N\right)>-\mu(N)-J^{\mu}\left(\psi^{-1}(N), N\right)=-\mu\left(\psi^{-1}(N)\right)=$ $-\left(\psi_{*} \mu\right)(N)$, and similarly, $b(1)>-\left(\psi_{*} \mu\right)\left(A_{1}\right)$.

Then we obtain a map $\chi: P \rightarrow \mathcal{D}_{\partial}^{c}\left(M_{1}\right)_{1}^{*}$ which satisfies conditions (1), (2) and (3) for these data. Condition (1) (i) assures that this map has a canonical extension by $i d$,

$$
\chi: P \rightarrow \mathcal{D}_{\partial \cup A_{2} \cup \cdots \cup A_{m}}^{c}(M)_{1}^{*},
$$

which also satisfies conditions (1), (3) (for $b(0), b(1))$ and

$$
\begin{aligned}
& \text { (i) } J^{\psi_{*} \mu}\left(\chi^{-1}(N), N\right)=b(0), \\
& \text { (ii) } J^{\psi_{*} \mu}\left(\chi^{-1}\left(A_{1}\right), A_{1}\right)=b(1) .
\end{aligned}
$$

Finally, we see that the map $\varphi: P \rightarrow \mathcal{D}_{\partial}^{c}(M)_{1}^{*}, \varphi_{p}=\chi_{p} \psi_{p}$, satisfies the required conditions.

(1) Since both $\psi$ and $\chi$ satisfy condition (1), so is the map $\varphi$.

(2) From condition $(2)_{2}$ (ii) it follows that

$$
\begin{aligned}
a(1) & =J^{\mu}\left(\psi^{-1}\left(A_{1}\right), A_{1}\right)+J^{\psi_{*} \mu}\left(\chi^{-1}\left(A_{1}\right), A_{1}\right) \\
& =J^{\mu}\left(\psi^{-1}\left(A_{1}\right), A_{1}\right)+J^{\mu}\left(\psi^{-1} \chi^{-1}\left(A_{1}\right), \psi^{-1} A_{1}\right)=J^{\mu}\left(\varphi^{-1}\left(A_{1}\right), A_{1}\right) .
\end{aligned}
$$

For $i=2, \cdots, m$, since $\chi=i d$ on $A_{i}$, condition $(2)_{1}$ (ii) implies that

$$
a(i)=J^{\mu}\left(\psi^{-1}\left(A_{i}\right), A_{i}\right)=J^{\mu}\left(\varphi^{-1}\left(A_{i}\right), A_{i}\right) .
$$

Condition (2)(i) follows from (2)(ii) and Remark 3.2(A).

(3) If $a_{p}(i)=0(i \in\{1, \cdots, m\})$, then we have (i) $\psi_{p}=i d_{M}$ and (ii) $b_{p}(0)=$ $b_{p}(1)=0$ so that $\chi_{p}=i d_{M}$. Thus $\varphi_{p}=i d_{M}$.

This completes the proof. 
3.3. Admissible sequences of diffeomorphisms. Suppose $M$ is a noncompact connected $C^{\infty} n$-manifold, possibly with boundary, and $d$ is any fixed metric on $\bar{M}$. Let $\mathcal{N}(M)$ denote the set of compact, connected $C^{\infty} n$-submanifold $N$ of $M$ such that each $C \in \mathcal{C}\left(N^{c}\right)$ is noncompact. (We assume that $\emptyset \in \mathcal{N}(M)$.) We set

$$
\begin{aligned}
\mathcal{N}^{(2)}(M)=\left\{(K, L) \in \mathcal{N}(M)^{2} \mid\right. & \text { (a) } K \subset \operatorname{Int}_{M} L \\
& \text { (b) } \left.L \cap A \text { is connected for each } A \in \mathcal{C}\left(K^{c}\right)\right\} .
\end{aligned}
$$

Note that (i) for any $N \in \mathcal{N}(M)$ the complementary region $N^{c}$ is a $C^{\infty} n$ submanifold of $M$ consisting of finitely many noncompact connected components and (ii) since $\operatorname{dim} E_{M}=0$, for any $K \in \mathcal{N}(M)$ and any $\varepsilon>0$ there exists an $L \in \mathcal{N}(M)$ such that $(K, L) \in \mathcal{N}^{(2)}(M)$ and $\operatorname{diam}_{d} B<\varepsilon\left(B \in \mathcal{C}\left(L^{c}\right)\right)$.

Let $P$ be a fixed topological space.

Definition 3.1. A sequence $\left(K_{k}, L_{k}, f^{k}, g^{k}\right)(k=1,2, \cdots)$ is said to be admissible if it satisfies the following conditions for each $k=1,2, \cdots$ :

(let $L_{0}=\emptyset$ and $f^{0}=g^{0} \equiv i d_{M}$ )

$(1)_{k} K_{k}, L_{k} \in \mathcal{N}(M)$ and $\left(L_{k-1}, K_{k}\right),\left(K_{k}, L_{k}\right) \in \mathcal{N}^{(2)}(M)$.

$(2)_{k} \quad$ (i) $f^{k}, g^{k}: P \rightarrow \mathcal{D}_{\partial}^{c}(M)_{1}^{*}$ are continuous maps.

(ii) $f^{k}=\varphi^{k} f^{k-1}$ and $g^{k}=\psi^{k} g^{k-1}$ for some continuous maps $\varphi^{k}: P \rightarrow$ $\mathcal{D}_{\partial \cup L_{k-1}}^{c}(M)_{1}^{*}$ and $\psi^{k}: P \rightarrow \mathcal{D}_{\partial \cup K_{k}}^{c}(M)_{1}^{*}$.

$(3)_{k} \quad$ (i) $f^{k}$ and $g^{k}$ have locally common compact support in $\operatorname{Int} M$.

(ii) $\left\{\left(f_{p}^{k}\right)^{-1}\right\}_{p}$ and $\left\{\left(g_{p}^{k}\right)^{-1}\right\}_{p}$ are equi-continuous with respect to $\left.d\right|_{M}$.

(4) $)_{k} \quad$ (i) $\quad \operatorname{diam} A \leq 2^{-k}, \quad \operatorname{diam}\left(g^{k-1}\right)^{-1}(A) \leq 2^{-k} \quad\left(A \in \mathcal{C}\left(K_{k}^{c}\right)\right)$.

(ii) $\operatorname{diam} B \leq 2^{-k}, \quad \operatorname{diam}\left(f^{k}\right)^{-1}(B) \leq 2^{-k} \quad\left(B \in \mathcal{C}\left(L_{k}^{c}\right)\right)$.

Lemma 3.4. Suppose $\left(K_{k}, L_{k}, f^{k}, g^{k}\right)_{k}$ is an admissible sequence.

(1) The sequences $\left(f^{k}\right)_{k}$ and $\left(g^{k}\right)_{k}$ converge $\left.d\right|_{M}$-uniformly to some continuous maps $f, g: P \rightarrow \mathcal{D}_{\partial}(M)_{1}$, respectively.

(2) $\left.f^{-1}\right|_{L^{k}}=\left.\left(f^{k}\right)^{-1}\right|_{L^{k}},\left.\quad g^{-1}\right|_{K^{k}}=\left.\left(g^{k-1}\right)^{-1}\right|_{K^{k}} \quad(k \geq 1)$.

Proof. We verify the statements for the map $f$. The argument for the map $g$ is completely the same. Note that the metric $d$ on $\bar{M}$ induces the sup-metric $d$ on $\operatorname{Map}(\bar{M})$ (and on $\operatorname{Map}(M)$ ).

[1] The sequence $\left(f^{k}\right)_{k}$ has the following properties:

(i) $d\left(f^{k}, f^{k+1}\right) \leq 2^{-k}, d\left(\left(f^{k}\right)^{-1},\left(f^{k+1}\right)^{-1}\right) \leq 2^{-k} \quad(k \geq 1)$,

(ii) $\left.\left(f^{\ell}\right)^{-1}\right|_{L^{k}}=\left.\left(f^{k}\right)^{-1}\right|_{L^{k}} \quad(\ell \geq k)$.

In fact, from $(2)_{k}$ and $(4)_{k}$ it follows that

(a) $\left.\varphi^{k+1}\right|_{L_{k}}=i d_{L_{k}} \quad$ and $\quad\left(\right.$ b) $\varphi^{k+1}(B)=B, \operatorname{dim} B \leq 2^{-k}\left(B \in \mathcal{C}\left(L_{k}^{c}\right)\right)$.

This implies that $d\left(\varphi^{k+1}, i d_{M}\right) \leq 2^{-k}$ and $d\left(f^{k}, f^{k+1}\right) \leq 2^{-k}$. On the other hand, since (c) $\left.\left(\varphi^{k+1}\right)^{-1}\right|_{L^{k}}=i d_{L^{k}}$ and (d) $\left(\varphi^{k+1}\right)^{-1}(B)=B\left(B \in \mathcal{C}\left(L_{k}^{c}\right)\right)$, it follows that $\left.\left(f^{k+1}\right)^{-1}\right|_{L^{k}}=\left.\left(f^{k}\right)^{-1}\right|_{L^{k}}$ and $\left(f^{k+1}\right)^{-1}(B)=\left(f^{k}\right)^{-1}(B)$, whose diameter $\leq 2^{-k}$ by $(4)_{k}$. This implies $d\left(\left(f^{k}\right)^{-1},\left(f^{k+1}\right)^{-1}\right) \leq 2^{-k}$. By $(2)_{k}$ (ii), for $\ell \geq k+1$ we have $\left.\varphi^{\ell}\right|_{L^{k}}=i d_{L^{k}}$ and

$$
\left.\left(f^{\ell}\right)^{-1}\right|_{L^{k}}=\left.\left(\varphi^{\ell} \cdots \varphi^{k+1} f^{k}\right)^{-1}\right|_{L^{k}}=\left.\left(f^{k}\right)^{-1}\left(\varphi^{\ell} \cdots \varphi^{k+1}\right)^{-1}\right|_{L^{k}}=\left.\left(f^{k}\right)^{-1}\right|_{L^{k}} .
$$


[2] There exists a canonical extension map $\eta: \mathcal{D}_{\partial}(M)_{1} \rightarrow \mathcal{H}_{\partial \cup E_{M}}(\bar{M})$. By [1] (i) the maps $\bar{f}^{k}=\eta f^{k}(k \geq 1)$ satisfy the following conditions:

$$
d\left(\bar{f}^{k}, \bar{f}^{k+1}\right) \leq 2^{-k} \quad \text { and } \quad d\left(\left(\bar{f}^{k}\right)^{-1},\left(\bar{f}^{k+1}\right)^{-1}\right) \leq 2^{-k} \quad(k \geq 1) .
$$

Since $\bar{M}$ is compact, the sequences $\left(\bar{f}^{k}\right)_{k}$ and $\left(\left(\bar{f}^{k}\right)^{-1}\right)_{k}$ converge $d$-uniformly to some continuous maps $\bar{f}, \bar{f}^{\prime}: P \rightarrow \operatorname{Map}_{\partial \cup E_{M}}(\bar{M})$. Since

$$
\bar{f}^{\prime} \bar{f}=\lim _{k \rightarrow \infty}\left(\bar{f}^{k}\right)^{-1} \bar{f}^{k} \equiv i d_{\bar{M}} \quad \text { and } \quad \bar{f}^{\prime}=\lim _{k \rightarrow \infty} \bar{f}^{k}\left(\bar{f}^{k}\right)^{-1} \equiv i d_{\bar{M}},
$$

it follows that $\bar{f}_{p} \in \mathcal{H}_{\partial \cup E_{M}}(\bar{M})$ and $f_{p}=\left.\bar{f}_{p}\right|_{M} \in \mathcal{H}_{\partial}(M)$ for each $p \in P$.

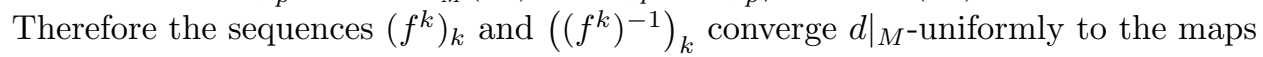
$f=\left.\bar{f}\right|_{M}, f^{-1}: P \rightarrow \mathcal{H}_{\partial}(M)$. In [1] (ii), tending $\ell \rightarrow \infty$, we have condition (2) (i.e., $\left.\left.f^{-1}\right|_{L^{k}}=\left.\left(f^{k}\right)^{-1}\right|_{L^{k}}(k \geq 1)\right)$.

[3] Since $\left(f_{p}^{k}\right)^{-1} \in \mathcal{D}_{\partial}(M)$ and $\left.f_{p}^{-1}\right|_{L^{k}}$ is a $C^{\infty}$-embedding for any $k \geq 1$, we have $f_{p}^{-1} \in \mathcal{D}_{\partial}(M)$. To see that $f_{p} \in \mathcal{D}_{\partial}(M)_{1}$, we have to construct a path $F_{p}(t) \in$ $\mathcal{D}_{\partial}(M)(t \in[0,1])$ with $F_{p}(0)=i d_{M}$ and $F_{p}(1)=f_{p}$. Since $\varphi_{p}^{k} \in \mathcal{D}_{\partial \cup L^{k-1}}^{c}(M)_{1}^{*}$, there exists a path $\Phi_{p}^{k}(t) \in \mathcal{D}_{\partial \cup L^{k-1}}^{c}(M)(k-1 \leq t \leq k)$ such that $\Phi_{p}^{k}(k-1)=i d_{M}$ and $\Phi_{p}^{k}(k)=\varphi_{p}^{k}$. Replacing $[0,1]$ by $[0, \infty]$, we define $F_{p}(t) \in \mathcal{D}_{\partial}(M)(t \in[0, \infty])$ by

$$
F_{p}(t)= \begin{cases}\Phi_{p}^{k}(t) f_{p}^{k-1} & (k \geq 1, k-1 \leq t \leq k), \\ f_{p} & (t=\infty) .\end{cases}
$$

Obviously $F_{p}(t)$ is continuous on $[0, \infty)$. Since $\left.F_{p}(t)^{-1}\right|_{L^{k}}=\left.\left(f_{p}^{k}\right)^{-1}\right|_{L^{k}}(k \leq t \leq \infty)$, it follows that $F_{p}(t)^{-1} \in \mathcal{D}(M)$ is continuous at $t=\infty$. Hence $F_{p}(t)$ is also continuous at $t=\infty$.

[4] Since the maps $\left(f^{k}\right)^{-1}: P \rightarrow \mathcal{D}_{\partial}(M)_{1}(k \geq 1)$ are continuous, by condition (2) and the definition of the compact-open $C^{\infty}$-topology, it follows that the map $f^{-1}: P \rightarrow \mathcal{D}_{\partial}(M)_{1}$ is continuous. This implies the continuity of the map $f: P \rightarrow$ $\mathcal{D}_{\partial}(M)_{1}$. This completes the proof.

Suppose $M$ is a noncompact, connected oriented $C^{\infty} n$-manifold, possibly with boundary, and $L_{i}(i \geq 0)$ is a sequence of compact $n$-submanifolds of $M$ such that $L_{0}=\emptyset, \bigcup_{i} L_{i}=M$ and $L_{i-1} \subset \operatorname{Int}_{M} L_{i} \quad(i \geq 1)$. We set $S=\bigcup_{i} \operatorname{Fr}_{M} L_{i}$ and $\mathcal{L}=\bigcup_{i} \mathcal{C}\left(\operatorname{cl}\left(L_{i}-L_{i-1}\right)\right)$.

Lemma 3.5. Suppose $\mu, \nu: P \rightarrow \mathcal{V}^{+}(M)$ are continuous maps such that $\mu(N)$ $=\nu(N)(N \in \mathcal{L})$. Then there exists a map $\chi: P \rightarrow \mathcal{D}_{\partial}(M, S)_{1}$ such that

(1) $\chi_{*} \mu=\nu$,

(2) $\chi(N)=N \quad(N \in \mathcal{L})$,

(3) if $p \in P$ and $\mu_{p}=\nu_{p}$, then $\chi_{p}=i d_{M}$.

Proof. Choose a product collar $E^{1}=\partial M \times[0,1]$ of $\partial M$ in $M$ and a product bicollar $E^{2}=S \times[-1,1]$ of $S$ in $M$ such that $E^{1}$ intersects with $E^{2}$ orthogonally so that

$$
E^{1} \cap E^{2}=\partial S \times[0,1] \times[-1,1]
$$

and

$$
E^{1}[0, a] \cap E^{2}[-b, b]=\partial S \times[0, a] \times[-b, b] \quad(a, b \in[0,1]) .
$$

By Lemma 2.3 (2) there exist maps

$$
\chi^{1}: P \rightarrow \mathcal{D}_{\partial \cup\left(M \backslash E^{1}\right)}(M)_{1} \quad \text { and } \quad \varepsilon^{1}: P \rightarrow C^{\infty}(\partial M,(0,1))
$$


such that for each $p \in P$,

(i) $\left(\chi_{p}^{1}\right)_{*} \mu_{p}=\nu_{p}$ on $E^{1}\left[0, \varepsilon_{p}^{1}\right]$,

(ii) $\chi_{p}^{1}\left(E_{x}^{1}\right)=E_{x}^{1}(x \in \partial M)$,

(iii) if $\mu_{p}=\nu_{p}$, then $\chi_{p}^{1}=i d_{M}$.

From (ii) it follows that $\chi_{p}^{1} \in \mathcal{D}_{\partial}(M ; S)_{1}$ and $\chi_{p}^{1}(N)=N(N \in \mathcal{L})$. Thus we obtain maps

$$
\chi_{p}^{1}: P \rightarrow \mathcal{D}_{\partial}(M ; S)_{1} \quad \text { and } \quad \mu^{1}=\chi_{*}^{1} \mu: P \rightarrow \mathcal{V}^{+}(M), \mu_{p}^{1}=\left(\chi_{p}^{1}\right)_{*} \mu_{p} .
$$

By Lemma 2.3 (1) there exist maps

$$
\chi^{2}: P \rightarrow \mathcal{D}_{S \cup\left(M \backslash E^{2}\right)}(M)_{1} \quad \text { and } \quad \varepsilon^{2}: P \rightarrow C^{\infty}(S,(0,1))
$$

such that for each $p \in P$,

(i) $)^{\prime}\left(\chi_{p}^{2}\right)_{*} \mu_{p}^{1}=\nu_{p}$ on $E^{2}\left[-\varepsilon_{p}^{2}, \varepsilon_{p}^{2}\right]$,

(ii)' for each $x \in S$

(a) $\chi_{p}^{2}\left(\left(E^{2}\right)_{x}^{ \pm}\right)=\left(E^{2}\right)_{x}^{ \pm}$

(b) if $\mu_{p}^{1}=\nu_{p}$ on $\left(E^{2}\right)_{x}^{ \pm}$, then $\chi_{p}^{2}=i d$ on $\left(E^{2}\right)_{x}^{ \pm}$.

From (ii) ${ }^{\prime}$ it follows that $\chi_{p}^{2} \in \mathcal{D}_{\partial \cup S \cup\left(M \backslash E^{2}\right)}(M)_{1}$ and $\chi_{p}^{2}(N)=N(N \in \mathcal{L})$. Thus we obtain the maps

$$
\chi_{p}^{2}: P \rightarrow \mathcal{D}_{\partial}(M ; S)_{1} \quad \text { and } \quad \mu^{2}=\chi_{*}^{2} \mu^{1}: P \rightarrow \mathcal{V}^{+}(M), \mu_{p}^{2}=\left(\chi_{p}^{2}\right)_{*} \mu_{p}^{1} .
$$

For each $N \in \mathcal{L}$, let $\partial_{-} N=\partial N \cap \partial M$ and $\partial_{+} N=\operatorname{Fr}_{M} N=\partial N \cap S$. Then

$$
E^{N}=\left(E^{1} \cup E^{2}\right) \cap N=\left(E_{\partial_{+} N}^{2} \cap N\right) \cup E_{\partial_{-} N}^{1}
$$

is a collar of $\partial N$ in $N$. Consider the continuous maps $\left.\mu^{2}\right|_{N},\left.\nu\right|_{N}: P \rightarrow \mathcal{V}^{+}(N)$ and

$$
\varepsilon^{N}: P \rightarrow(0,1 / 2), \quad \varepsilon_{p}^{N}=\frac{1}{2} \min \left\{\left.\min \varepsilon_{p}^{1}\right|_{\partial_{-} N},\left.\min \varepsilon_{p}^{2}\right|_{\partial_{+} N}\right\} .
$$

For each $p \in P$ it is seen that

$$
\mu_{p}^{2}(N)=\nu_{p}(N) \quad \text { and }\left.\quad \mu_{p}^{2}\right|_{N}=\left.\nu_{p}\right|_{N} \text { on } E^{N}\left[0,2 \varepsilon_{p}^{N}\right] .
$$

In fact, since $\chi_{p}^{2}(N)=\chi_{p}^{1}(N)=N$, it follows that $\mu_{p}^{2}(N)=\mu_{p}^{1}(N)=\mu_{p}(N)=$ $\nu_{p}(N)$. By (i) ${ }^{\prime}$ and (i) we have $\mu_{p}^{2}=\nu_{p}$ on $E_{\partial_{+} N}^{2}\left[-2 \varepsilon_{p}^{N}, 2 \varepsilon_{p}^{N}\right]$ and $\mu_{p}^{1}=\nu_{p}$ on $E_{\partial_{-} N}^{1}\left[0,2 \varepsilon_{p}^{N}\right]$. The latter and (ii)' (b) imply that $\chi_{p}^{2}=i d$ on $E_{\partial_{-} N}^{1}\left[0,2 \varepsilon_{p}^{N}\right]$, and so $\mu_{p}^{2}=\mu_{p}^{1}=\nu_{p}$ on $E_{\partial_{-} N}^{1}\left[0,2 \varepsilon_{p}^{N}\right]$.

Then, Theorem 2.1 yields a map $\chi^{N}: P \rightarrow \mathcal{D}_{\partial}(N)_{1}$ such that for each $p \in P$

(i) $)^{\prime \prime}\left(\chi_{p}^{N}\right)_{*}\left(\left.\mu_{p}^{2}\right|_{N}\right)=\left.\nu_{p}\right|_{N}$,

(ii)" $\chi_{p}^{N}=i d$ on $E^{N}\left[0, \varepsilon_{p}^{N}\right] \quad\left(\chi_{p}^{N}\right.$ is isotopic to $i d_{N}$ relative to $\left.E^{N}\left[0, \varepsilon_{p}^{N}\right]\right)$,

(iii) $^{\prime \prime}$ if $\left.\mu_{p}^{2}\right|_{N}=\left.\nu_{p}\right|_{N}$, then $\chi_{p}^{N}=i d_{N}$.

Define a map $\chi^{3}: P \rightarrow \mathcal{D}_{\partial \cup S}(M)_{1}$ by $\left.\chi_{p}^{3}\right|_{N}=\chi_{p}^{N}(N \in \mathcal{L})$. Then we have $\left(\chi_{p}^{3}\right)_{*} \mu_{p}^{2}=\nu_{p}(p \in P)$.

Finally, the required map $\chi$ is defined by the composition

$$
\chi: P \rightarrow \mathcal{D}_{\partial}(M, S)_{1}, \quad \chi_{p}=\chi_{p}^{3} \chi_{p}^{2} \chi_{p}^{1} \quad(p \in P) .
$$

If $\mu_{p}=\nu_{p}$, then $\chi_{p}^{1}=i d_{M}$ and $\mu_{p}^{1}=\nu_{p}$. In turn, this implies that $\chi_{p}^{2}=i d_{M}$ and $\mu_{p}^{2}=\mu_{p}^{1}=\nu_{p}$, so that $\chi_{p}^{3}=i d_{M}$ and hence $\chi_{p}=i d_{M}$. 
3.4. Main deformation theorem. Suppose $M$ is a noncompact connected oriented $C^{\infty} n$-manifold, possibly with boundary, $d$ is a fixed metric on $\bar{M}$ and $\mu, \nu: P \rightarrow \mathcal{V}^{+}(M)$ are continuous maps. Let $\mathcal{F}(M)$ denote the class of connected $C^{\infty} n$-submanifold $N$ of $M$ such that $\operatorname{Fr}_{M} N$ is compact and set $\mathcal{F}_{c}(M)=$ $\{N \in \mathcal{F}(M) \mid N$ : compact $\}$. Let $C^{0}(P)$ denote the set of all continuous functions $\alpha: P \rightarrow \mathbb{R}$ and $\mathcal{D}$ denote the collection of all continuous maps $f: P \rightarrow \mathcal{D}_{\partial}(M)_{1}$.

Assumption 3.1. Suppose a subclass $\mathcal{F}$ of $\mathcal{F}(M)$ and a map $a: \mathcal{D}^{2} \times \mathcal{F} \rightarrow C^{0}(P)$ satisfy the following conditions:

$\left(*_{0}\right) \mathcal{F}_{c}(M) \subset \mathcal{F}$ and $\mu(A)=\nu(A)=\infty(A \in \mathcal{F}(M)-\mathcal{F})$.

$\left(*_{1}\right) a(f, g ; C) \in\left(-\mu\left(f^{-1}(C)\right), \nu\left(g^{-1}(C)\right)\right) \quad(f, g \in \mathcal{D}, C \in \mathcal{F})$.

$\left(*_{2}\right) a\left(f_{2}, g ; C\right)=a\left(f_{1}, g ; C\right)+J^{\mu}\left(f_{1}^{-1}(C), f_{2}^{-1}(C)\right), a\left(f, g_{2} ; C\right)=a\left(f, g_{1} ; C\right)-$ $J^{\nu}\left(g_{1}^{-1}(C), g_{2}^{-1}(C)\right)\left(f, f_{1}, f_{2}, g, g_{1}, g_{2} \in \mathcal{D}, C \in \mathcal{F}\right)$.

(*3) If $(K, L) \in \mathcal{N}^{(2)}(M), A \in \mathcal{C}\left(K^{c}\right)$ and $\mathcal{C}\left(A \cap L^{c}\right) \subset \mathcal{F}$, then $A \in \mathcal{F}$ and

$$
a(f, g ; A \cap L)+\sum_{B \in \mathcal{C}\left(A \cap L^{c}\right)} a(f, g ; B)=a(f, g ; A) \quad(f, g \in \mathcal{D}) .
$$

$\left(*_{4}\right)$ If $M \in \mathcal{F}$, then $a\left(i d_{M}, i d_{M} ; M\right)=0$.

Remark 3.3. From $\left(*_{2}\right)$ it follows that for $p \in P$

(i) $a_{p}\left(f_{1}, g_{1} ; C\right)=a_{p}\left(f_{2}, g_{2} ; C\right)$ if $\left(f_{1}\right)_{p}^{-1}(C)=\left(f_{2}\right)_{p}^{-1}(C)$ and $\left(g_{1}\right)_{p}^{-1}(C)$ $=\left(g_{2}\right)_{p}^{-1}(C)$ (for example, $\left(f_{1}\right)_{p}=\left(f_{2}\right)_{p}$ and $\left.\left(g_{1}\right)_{p}=\left(g_{2}\right)_{p}\right)$,

(ii) $a_{p}(\varphi f, g ; C)=a_{p}(f, g ; C)$ if $\varphi \in \mathcal{D}$ and $\varphi_{p}=i d$ on $C$.

See Section 4 for explicit examples of $(a, \mathcal{F})$.

Recall the defining conditions $(1)_{k} \sim(4)_{k}$ for admissible sequences in Definition 3.1 .

Theorem 3.1. [1] There exists an admissible sequence $\left(K_{k}, L_{k}, f^{k}, g^{k}\right)(k=1,2$, $\cdots)\left(L^{0}=\emptyset, f^{0}=g^{0} \equiv i d_{M}\right)$ which satisfies the following additional conditions:

$$
\begin{array}{lll}
\text { (i) } & a\left(f^{k}, g^{k-1} ; C\right)=0 & \left(C \in \mathcal{C}\left(\operatorname{cl}\left(K_{k}-L_{k-1}\right)\right) \cup\left(\mathcal{C}\left(K_{k}^{c}\right) \cap \mathcal{F}\right)\right), \\
\text { (ii) } & a\left(f^{k}, g^{k} ; C\right)=0 & \left(C \in \mathcal{C}\left(\operatorname{cl}\left(L_{k}-K_{k}\right)\right) \cup\left(\mathcal{C}\left(L_{k}^{c}\right) \cap \mathcal{F}\right)\right) .
\end{array}
$$

$(6)_{k}$ If $p \in P$ and $a_{p}\left(i d_{M}, i d_{M} ; C\right)=0(C \in \mathcal{F})$, then $f_{p}^{k}=g_{p}^{k}=i d_{M}$.

[2] The sequences $\left(f^{k}\right)_{k},\left(g^{k}\right)_{k}$ converge $\left.d\right|_{M}$-uniformly to some maps $f, g: P \rightarrow$ $\mathcal{D}_{\partial}(M)_{1}$, respectively. The maps $f$ and $g$ satisfy the following conditions:

(1) $\left.f^{-1}\right|_{L_{k}}=\left.\left(f^{k}\right)^{-1}\right|_{L_{k}},\left.\quad g^{-1}\right|_{K_{k}}=\left.\left(g^{k-1}\right)^{-1}\right|_{K_{k}} \quad(k \geq 1)$.

(2) $a(f, g ; C)=0$ for any $C \in \mathcal{F}$ with $C \in \mathcal{C}\left(K_{k}^{c}\right) \cup \mathcal{C}\left(L_{k}^{c}\right) \cup \mathcal{C}\left(\operatorname{cl}\left(K_{k}-L_{k-1}\right)\right) \cup$ $\mathcal{C}\left(\operatorname{cl}\left(L_{k}-K_{k}\right)\right)$ for some $k \geq 1$.

(3) If $p \in P$ and $a_{p}\left(i d_{M}, i d_{M} ; C\right)=0(C \in \mathcal{F})$, then $f_{p}=g_{p}=i d_{M}$.

Proof. [1] Suppose $k \geq 1$ and assume that we have constructed $\left(K_{i}, L_{i}, f^{i}, g^{i}\right)$ $(i=1, \cdots, k-1)$ which satisfy conditions $(1)_{i} \sim(6)_{i}(i=1, \cdots, k-1)$. The tuple $\left(K_{k}, L_{k}, f^{k}, g^{k}\right)$ is constructed as follows:

$K_{k}$ : Since $\left\{\left(g_{p}^{k-1}\right)^{-1}\right\}_{p}$ is equi-continuous with respect to $\left.d\right|_{M}$, we can find $K_{k} \in \mathcal{N}(M)$ which satisfies $(1)_{k}$ and $(4)_{k}(\mathrm{i})$.

$f^{k}:$ For each $B \in \mathcal{C}\left(L_{k-1}^{c}\right)$ we construct a map $\varphi^{B}: P \rightarrow \mathcal{D}_{\partial \cup B^{c}}^{c}(M)_{1}^{*}$ which satisfies the following conditions:

$(3)_{k}^{\prime} \quad$ (i) $\varphi^{B}$ has locally common compact support in $\operatorname{Int} M$.

(ii) $\left\{\left(\varphi_{p}^{B}\right)^{-1}\right\}_{p}$ is equi-continuous with respect to $\left.d\right|_{M}$. 
$(5)_{k}^{\prime} \quad$ (i) $a\left(\varphi^{B} f^{k-1}, g^{k-1} ; B \cap K_{k}\right)=0$

(ii) $a\left(\varphi^{B} f^{k-1}, g^{k-1} ; A\right)=0\left(A \in \mathcal{C}\left(B \cap K_{k}^{c}\right) \cap \mathcal{F}\right)$.

$(6)_{k}^{\prime}$ If $p \in P$ and $a_{p}\left(i d_{M}, i d_{M} ; C\right)=0(C \in \mathcal{F})$, then $\varphi_{p}^{B}=i d_{M}$.

Consider the decomposition of $B$ and the associated functions in $C^{0}(P)$ defined by

(i) $B=N \cup\left(\bigcup_{i=1}^{\ell} A_{i}\right) \cup\left(\bigcup_{j=1}^{m} A_{j}^{\prime}\right)(\ell, m \geq 0, \ell+m \geq 1)$, where $N=A_{0}=$ $B \cap K_{k}, \mathcal{C}\left(B \cap K_{k}^{c}\right) \cap \mathcal{F}=\left\{A_{1}, \cdots, A_{\ell}\right\}, \mathcal{C}\left(B \cap K_{k}^{c}\right)-\mathcal{F}=\left\{A_{1}^{\prime}, \cdots, A_{m}^{\prime}\right\}$.

(ii) $a(i)=a\left(f^{k-1}, g^{k-1} ; A_{i}\right)(i=0,1, \cdots, \ell), b(j)=-\frac{1}{m} \sum_{i=0}^{\ell} a(i)(j=1, \cdots, m)$.

In order to apply Lemma 3.3 to these data and $\left.\left(f_{*}^{k-1} \mu\right)\right|_{B}$, we have to check the following conditions:

(a) $a(0)+\sum_{i=1}^{\ell} a(i)+\sum_{j=1}^{m} b(j)=0$,

(b) $a(i)>-\mu\left(\left(f^{k-1}\right)^{-1}\left(A_{i}\right)\right) \quad(i=0,1, \cdots, \ell)$,

$b(j)>-\mu\left(\left(f^{k-1}\right)^{-1}\left(A_{j}^{\prime}\right)\right) \quad(j=1, \cdots, m)$.

For (a), if $m \geq 1$, then this follows from the definition of $b(j)$. If $m=0$, then from $\left(*_{3}\right)$ and $(5)_{k-1}\left(\right.$ ii) $\left(\left(*_{4}\right)\right.$ for $\left.k=1\right)$ it follows that $B \in \mathcal{F}$ and $\sum_{i=0}^{\ell} a(i)=$ $a\left(f^{k-1}, g^{k-1} ; B\right)=0$.

For (b), the assertion for $a(i)$ follows from $\left(*_{1}\right)$. Since $A_{j}^{\prime} \notin \mathcal{F}$, from $\left(*_{0}\right)$ it follows that $\mu\left(A_{j}^{\prime}\right)=\infty$. Since each $f_{p}^{k-1}$ has a compact support, we also have $\mu\left(\left(f^{k-1}\right)^{-1}\left(A_{j}^{\prime}\right)\right)=\infty$, which obviously implies the assertion for $b(j)$.

Now Lemma 3.3 can be applied to yield a map $\varphi^{B}: P \rightarrow \mathcal{D}_{\partial \cup B^{c}}^{c}(M)_{1}^{*}$ which satisfies $(3)_{k}^{\prime}$ and the following conditions:

$(5)_{k}^{\prime \prime} \quad J^{f^{k-1} \mu}\left(\left(\varphi^{B}\right)^{-1}\left(A_{i}\right), A_{i}\right)=a(i)(i=0,1, \cdots, \ell), J^{f^{k-1} \mu}\left(\left(\varphi^{B}\right)^{-1}\left(A_{j}^{\prime}\right), A_{j}^{\prime}\right)=$ $b(j)(j=1, \cdots, m)$.

$(6)_{k}^{\prime \prime}$ If $p \in P$ and $a_{p}(i)=0(i=0,1, \cdots, \ell)$ (so that $b_{p}(j)=0(j=1, \cdots, m)$, then $\varphi_{p}^{B}=i d_{M}$.

We note that conditions $(5)_{k}^{\prime \prime}$ and $(6)_{k}^{\prime \prime}$ imply $(5)_{k}^{\prime}$ and $(6)_{k}^{\prime}$, respectively. In fact, by $\left(*_{2}\right)$ and Lemma 2.1 condition $(5)_{k}^{\prime \prime}$ implies that

$$
\begin{aligned}
a\left(\varphi^{B} f^{k-1}, g^{k-1} ; A_{i}\right) & =a\left(f^{k-1}, g^{k-1} ; A_{i}\right)+J^{\mu}\left(\left(f^{k-1}\right)^{-1}\left(A_{i}\right),\left(\varphi^{B} f^{k-1}\right)^{-1}\left(A_{i}\right)\right) \\
& =a(i)-J^{f^{k-1} \mu}\left(\left(\varphi^{B}\right)^{-1}\left(A_{i}\right), A_{i}\right)=0 \quad(i=0,1, \cdots, \ell) .
\end{aligned}
$$

If $p \in P$ and $a_{p}\left(i d_{M}, i d_{M} ; C\right)=0(C \in \mathcal{F})$, then from $(6)_{k-1}$ and Remark 3.3 (i) it follows that

and

$$
f_{p}^{k-1}=g_{p}^{k-1}=i d_{M}
$$

$$
a_{p}(i)=a_{p}\left(f^{k-1}, g^{k-1} ; A_{i}\right)=a_{p}\left(i d_{M}, i d_{M} ; A_{i}\right)=0 \quad(i=0,1, \cdots, \ell),
$$

and from $(6)_{k}^{\prime \prime}$ follows $\varphi_{p}^{B}=i d_{M}$.

Finally, we define a map $\varphi^{k}: P \rightarrow \mathcal{D}_{\partial \cup L_{k-1}}^{c}(M)_{1}^{*}$ by

$$
\varphi_{p}^{k}=\varphi_{p}^{B_{1}} \cdots \varphi_{p}^{B_{r}} \quad(p \in P) \text {, where } \mathcal{C}\left(L_{k-1}^{c}\right)=\left\{B_{1}, \cdots, B_{r}\right\} \text { is any ordering. }
$$

Then the required map $f^{k}: P \rightarrow \mathcal{D}_{\partial}^{c}(M)_{1}^{*}$ is defined by $f^{k}=\varphi^{k} f^{k-1}$. The conditions $(3)_{k}$ and $(6)_{k}$ follow from $(3)_{k-1},(3)_{k}^{\prime}$ and $(6)_{k-1},(6)_{k}^{\prime}$ respectively, while condition $(5)_{k}$ follows from $(5)_{k}^{\prime}$ and Remark 3.3 (ii).

$L_{k}$ : Since $\left\{\left(f_{p}^{k}\right)^{-1}\right\}_{p}$ is equi-continuous, we can find $L_{k} \in \mathcal{N}(M)$ which satisfies $(1)_{k}$ and $(4)_{k}(\mathrm{ii})$. 
$g^{k}$ : The map $\psi^{k}$ and $g^{k}$ are obtained by the same arguments, using Lemma 3.3 and Remark $3.2(\mathrm{~B})$.

[2] The existence of the limit maps $f$ and $g$ and condition (1) follow from Lemma 3.4

(2) For each $k \geq 1$

(i) since $f^{-1}=\left(f^{k}\right)^{-1}$ and $g^{-1}=\left(g^{k-1}\right)^{-1}$ on $K_{k}$, for any $C \in\left(\mathcal{C}\left(K_{k}^{c}\right) \cap\right.$ $\mathcal{F}) \cup \mathcal{C}\left(\operatorname{cl}\left(K_{k}-L_{k-1}\right)\right)$ it follows that $f^{-1}(C)=\left(f^{k}\right)^{-1}(C), g^{-1}(C)=$ $\left(g^{k-1}\right)^{-1}(C)$ and so $a(f, g ; C)=a\left(f^{k}, g^{k-1} ; C\right)=0$ by Remark 3.3 (i) and $(5)_{k}(\mathrm{i})$,

(ii) since $f^{-1}=\left(f^{k}\right)^{-1}$ and $g^{-1}=\left(g^{k}\right)^{-1}$ on $L_{k}$, for any $C \in\left(\mathcal{C}\left(L_{k}^{c}\right) \cap\right.$ $\mathcal{F}) \cup \mathcal{C}\left(\operatorname{cl}\left(L_{k}-K_{k}\right)\right)$ it follows that $f^{-1}(C)=\left(f^{k}\right)^{-1}(C), g^{-1}(C)=$ $\left(g^{k}\right)^{-1}(C)$ and hence $a(f, g ; C)=a\left(f^{k}, g^{k} ; C\right)=0$ by Remark 3.3(i) and $(5)_{k}$ (ii).

(3) The assertion follows from $(6)_{k}$.

\section{Proof of Theorems 1.1 And 1.2}

In this section we apply Theorem 3.1 to deduce Theorems 1.1 and 1.2. Suppose $M$ is a noncompact connected oriented $C^{\infty} n$-manifold, possibly with boundary, and $d$ is a fixed metric on $\bar{M}$.

4.1. Proof of Theorem 1.1. Suppose $F$ is an open subset of $E_{M}$ and $\mu, \nu: P \rightarrow$ $\mathcal{V}^{+}(M ; F)_{e w}$ are continuous maps with $\mu_{p}(M)=\nu_{p}(M)(p \in P)$.

Definition 4.1. We define $(\mathcal{F}, a)$ as follows:

(i) $\mathcal{F}=\left\{C \in \mathcal{F}(M) \mid E_{C} \subset F\right\}$,

(ii) $a: \mathcal{D}^{2} \times \mathcal{F} \rightarrow C^{0}(P)$ :

$$
a(f, g ; C)=\nu\left(g^{-1}(C)\right)-\mu\left(f^{-1}(C)\right)=\left(g_{*} \nu\right)(C)-\left(f_{*} \mu\right)(C) .
$$

Remark 4.1. For $C \in \mathcal{F}(M)$ and $f \in \mathcal{D}$ we have

(a) $C \in \mathcal{F}$ iff $\mu(C)<\infty$ (or $\nu(C)<\infty$ ), since $E_{M}^{\mu_{p}}=E_{M}^{\nu_{p}}=F(p \in P)$.

(b) $E_{f^{-1}(C)}=E_{C}$, and so $C \in \mathcal{F}$ iff $f^{-1}(C) \in \mathcal{F}$, since $f_{p} \in \mathcal{D}_{E_{M}}(M)(p \in P)$.

Lemma 4.1. The map a is well-defined and $(\mathcal{F}, a)$ satisfies the conditions $\left(*_{0}\right) \sim$ $\left(*_{4}\right)$ in Assumption 3.1 .

Proof. Remark 4.1 implies that $\mu\left(f^{-1}(C)\right), \nu\left(g^{-1}(C)\right)<\infty$. Since $\mu\left(f^{-1}(C)\right)=$ $J^{\mu}\left(f^{-1}(C), C\right)+\mu(C)$, by Lemmas 2.2 and 2.4 the map $\mu\left(f^{-1}(C)\right): P \rightarrow \mathbb{R}$ is continuous. Similarly, the map $\nu\left(g^{-1}(C)\right): P \rightarrow \mathbb{R}$ is continuous. Thus, the map $a(f, g ; C): P \rightarrow \mathbb{R}$ is continuous and the map $a$ is well-defined. The conditions $\left(*_{0}\right) \sim\left(*_{4}\right)$ are verified as follows:

$\left(*_{0}\right)$ Obviously $\mathcal{F}_{c}(M) \subset \mathcal{F}$, and by Remark 4.1(a) we have $\mu(A)=\nu(A)=\infty$ $(A \in \mathcal{F}(M)-\mathcal{F})$.

$$
\left(*_{1}\right) a(f, g ; C)=\nu\left(g^{-1}(C)\right)-\mu\left(f^{-1}(C)\right) \in\left(-\mu\left(f^{-1}(C)\right), \nu\left(g^{-1}(C)\right)\right) .
$$


$\left(*_{2}\right)$ Since $\mu\left(f_{i}^{-1}(C)\right), \nu\left(g_{i}^{-1}(C)\right)<\infty(i=1,2)$, we have

$$
\begin{aligned}
& a\left(f_{1}, g ; C\right)+J^{\mu}\left(f_{1}^{-1}(C), f_{2}^{-1}(C)\right) \\
& \quad=\nu\left(g^{-1}(C)\right)-\mu\left(f_{1}^{-1}(C)\right)+\mu\left(f_{1}^{-1}(C)\right)-\mu\left(f_{2}^{-1}(C)\right) \\
& \quad=\nu\left(g^{-1}(C)\right)-\mu\left(f_{2}^{-1}(C)\right)=a\left(f_{2}, g ; C\right), \\
& a\left(f, g_{1} ; C\right)-J^{\nu}\left(g_{1}^{-1}(C), g_{2}^{-1}(C)\right) \\
& \quad=\nu\left(g_{1}^{-1}(C)\right)-\mu\left(f^{-1}(C)\right)-\left(\nu\left(g_{1}^{-1}(C)\right)-\nu\left(g_{2}^{-1}(C)\right)\right) \\
& \quad=\nu\left(g_{2}^{-1}(C)\right)-\mu\left(f^{-1}(C)\right)=a\left(f, g_{2} ; C\right) .
\end{aligned}
$$

$\left(*_{3}\right)$ If $(K, L) \in \mathcal{N}^{(2)}(M), A \in \mathcal{C}\left(K^{c}\right)$ and $\mathcal{C}\left(A \cap L^{c}\right) \subset \mathcal{F}$, then

(i) $\mu(A)=\mu(A \cap L)+\sum_{B \in \mathcal{C}\left(A \cap L^{c}\right)} \mu(B)<\infty$, so that $A \in \mathcal{F}$,

(ii) $a(f, g ; A \cap L)+\sum_{B \in \mathcal{C}\left(A \cap L^{c}\right)} a(f, g ; B)$

$$
\begin{aligned}
& =\nu\left(g^{-1}(A \cap L)\right)-\mu\left(f^{-1}(A \cap L)\right)+\sum_{B \in \mathcal{C}\left(A \cap L^{c}\right)}\left(\nu\left(g^{-1}(B)\right)-\mu\left(f^{-1}(B)\right)\right) \\
& =\nu\left(g^{-1}(A)\right)-\mu\left(f^{-1}(A)\right) \\
& =a(f, g ; A) .
\end{aligned}
$$

$\left(*_{4}\right)$ If $M \in \mathcal{F}$, then $a\left(i d_{M}, i d_{M} ; M\right)=\nu(M)-\mu(M)=0$ by the assumption on $\mu$ and $\nu$.

Proof of Theorem 1.1. By Theorem 3.1 we obtain an admissible sequence $\left(K_{k}, L_{k}, f^{k}, g^{k}\right)_{k \geq 1}$ and the limit maps $f, g: P \rightarrow \mathcal{D}_{\partial}(M)_{1}$ which satisfy the following conditions:

(1) $a(f, g ; C)=0$ (i.e., $\left.\left(f_{*} \mu\right)(C)=\left(g_{*} \nu\right)(C)\right)$ for any $C \in \mathcal{C}\left(c l\left(K_{k}-L_{k-1}\right)\right) \cup$ $\mathcal{C}\left(\operatorname{cl}\left(L_{k}-K_{k}\right)\right) \quad(k \geq 1)$.

(2) If $p \in P$ and $a_{p}\left(i d_{M}, i d_{M} ; C\right)=0(C \in \mathcal{F})$ (i.e., $\left.\mu_{p}=\nu_{p}\right)$, then $f_{p}=g_{p}=$ $i d_{M}$.

By condition (1) and Lemma 3.5 we obtain a map $\chi: P \rightarrow \mathcal{D}_{\partial}(M)_{1}$ such that

(i) $\chi_{*}\left(f_{*} \mu\right)=g_{*} \nu$,

(ii) if $p \in P$ and $\left(f_{p}\right)_{*} \mu_{p}=\left(g_{p}\right)_{*} \nu_{p}$, then $\chi_{p}=i d_{M}$.

Finally, the required map $h: P \rightarrow \mathcal{D}_{\partial}(M)_{1}$ is defined by $h_{p}=g_{p}^{-1} \chi_{p} f_{p}(p \in P)$.

Theorem 1.1 is equivalent to the following statement.

Theorem 1.1'. Suppose $P$ is any topological space and $\widetilde{\mu}: P \times[0,1] \rightarrow \mathcal{V}^{+}(M ; F)_{\text {ew }}$ is a homotopy with $\widetilde{\mu}_{p}(t)(M)=\widetilde{\mu}_{p}(0)(M)((p, t) \in P \times[0,1])$. Then there exists a homotopy $\widetilde{h}: P \times[0,1] \rightarrow \mathcal{D}_{\partial}(M)_{1}$ such that for each $(p, t) \in P \times[0,1]$,

(1) $\widetilde{h}_{p}(t)_{*} \widetilde{\mu}_{p}(0)=\widetilde{\mu}_{p}(t)$,

(2) $\widetilde{h}_{p}(0)=i d_{M}$,

(3) if $\widetilde{\mu}_{p}(t)=\widetilde{\mu}_{p}(0)$, then $\widetilde{h}_{p}(t)=i d_{M}$.

Proof. The homotopy $\widetilde{h}$ in Theorem $1.1^{\prime}$ is obtained by applying Thereom 1.1 to the maps $\widetilde{\mu}$ and $\widetilde{\mu}^{\prime}: P \times[0,1] \rightarrow \mathcal{V}^{+}(M ; F)_{e w}, \widetilde{\mu}^{\prime}(t)=\widetilde{\mu}(0)(t \in[0,1])$.

The maps $\mu, \nu: P \rightarrow \mathcal{V}^{+}(M ; F)_{\text {ew }}$ in Theorem 1.1 are connected by the affine homotopy $\widetilde{\mu}: P \times[0,1] \rightarrow \mathcal{V}^{+}(M ; F)_{e w}, \widetilde{\mu}(t)=(1-t) \mu+t \nu$. Theorem $1.1^{\prime}$ yields a homotopy $\widetilde{h}$ which traces $\widetilde{\mu}$, and the map $h=\widetilde{h}(1)$ satisfies the required conditions in Theorem 1.1.

Proof of Corollary 1.1(1). We apply Theorem 1.1 to the data: $P=\mathcal{V}^{+}(M ; m, F)$, the constant map $P \rightarrow \mathcal{V}^{+}(M ; F), \mu \mapsto \omega$ and the inclusion map $P \subset \mathcal{V}^{+}(M ; F)$. 
This yields a map $\sigma: P \rightarrow \mathcal{D}(M)_{1}$ such that $\sigma(\mu)_{*} \omega=\mu(\mu \in P)$ and $\sigma(\omega)=i d_{M}$. This means that the map $\sigma$ is the required section of $\pi_{\omega}$.

Corollary 1.1 (2) is a special case of the next corollary.

Corollary 4.1. Suppose $G$ is any subgroup of $\mathcal{D}^{+}(M ; F)$ with $\mathcal{D}_{\partial}(M)_{1} \subset G$. Then

(1) $(G, G \cap \mathcal{D}(M ; \omega)) \cong\left(\mathcal{V}^{+}(M ; m, F)_{e w},\{\omega\}\right) \times(G \cap \mathcal{D}(M ; \omega))$.

(2) $G \cap \mathcal{D}(M ; \omega)$ is a strong deformation retract of $G$.

Proof. (1) The required homeomorphism $\varphi: G \rightarrow \mathcal{V}^{+}(M ; m, F)_{\text {ew }} \times(G \cap \mathcal{D}(M ; \omega))$ is defined by $\varphi(h)=\left(\pi_{\omega}(h),\left(\sigma\left(\pi_{\omega}(h)\right)\right)^{-1} h\right)$. The inverse is given by $\varphi^{-1}(\mu, h)=$ $\sigma(\mu) h$.

(2) Since the singleton $\{\omega\}$ is a strong deformation retract of $\mathcal{V}^{+}(M ; m, F)_{\text {ew }}$, the conclusion follows from (1).

Remark 4.2. $\mathcal{D}_{\partial}(M)_{1} \cap \mathcal{D}(M ; \omega)=\mathcal{D}_{\partial}(M ; \omega)_{1}$.

Proof. By Corollary $4.1(2) \mathcal{D}_{\partial}(M)_{1} \cap \mathcal{D}(M ; \omega)$ is a strong deformation retract of $\mathcal{D}_{\partial}(M)_{1}$. Since the latter is path-connected, so is the former.

4.2. Proof of Theorem 1.2. Suppose $\mu: P \rightarrow \mathcal{V}^{+}(M)$ and $a: P \rightarrow \mathcal{S}(M)$ are continuous maps such that $a_{p} \in \mathcal{S}\left(M, \mu_{p}\right)(p \in P)$. (Let $\nu=\mu$.)

Definition 4.2. We define $(\mathcal{F}, \widetilde{a})$ as follows:

(i) $\mathcal{F}=\mathcal{F}(M)$,

(ii) $\widetilde{a}: \mathcal{D}^{2} \times \mathcal{F} \rightarrow C^{0}(P): \quad \widetilde{a}(f, g ; C)=a\left(E_{C}\right)-J^{\mu}\left(f^{-1}(C), g^{-1}(C)\right)$.

Lemma 4.2. The map $\widetilde{a}$ is well-defined and $(\mathcal{F}, \widetilde{a})$ satisfies the conditions $\left(*_{0}\right) \sim$ $\left(*_{4}\right)$ in Assumption 3.1 .

Proof. Lemma 2.2 implies that the function $J^{\mu}\left(f^{-1}(C), g^{-1}(C)\right): P \rightarrow \mathbb{R}$ is welldefined and continuous, while the continuity of the function $a\left(E_{C}\right): P \rightarrow \mathbb{R}$ follows from the definition of the weak topology of $\mathcal{S}(M)$. The conditions $\left(*_{0}\right) \sim\left(*_{4}\right)$ are verified as follows:

$\left(*_{0}\right)$ The assertion is trivial.

$\left(*_{1}\right)$ Let $(f, g, C) \in \mathcal{D}^{2} \times \mathcal{F}$ and $p \in P$. Since $f_{p}, g_{p} \in \mathcal{D}_{E_{M}}(M)$, we have $E_{C}=E_{f_{p}^{-1}(C)}=E_{g_{p}^{-1}(C)}$. If $E_{C} \subset E_{M}^{\mu_{p}}$, then (a) $a_{p}\left(E_{C}\right)=0$ since $a_{p} \in \mathcal{S}\left(M ; \mu_{p}\right)$ and (b) $\mu_{p}\left(f_{p}^{-1}(C)\right), \mu_{p}\left(g_{p}^{-1}(C)\right)<\infty$, so that

$$
\widetilde{a}_{p}(f, g ; C)=-J^{\mu_{p}}\left(f_{p}^{-1}(C), g_{p}^{-1}(C)\right)=\mu_{p}\left(g_{p}^{-1}(C)\right)-\mu_{p}\left(f_{p}^{-1}(C)\right) .
$$

If $E_{C} \not \subset E_{M}^{\mu_{p}}$, then $\mu_{p}\left(f_{p}^{-1}(C)\right)=\mu_{p}\left(g_{p}^{-1}(C)\right)=\infty$. In both cases, we have $\widetilde{a}_{p}(f, g ; C) \in\left(-\mu_{p}\left(f_{p}^{-1}(C)\right), \mu_{p}\left(g_{p}^{-1}(C)\right)\right)$.

$\left(*_{2}\right)$ By Lemma 2.1 it follows that

$$
\begin{aligned}
& \widetilde{a}\left(f_{1}, g ; C\right)+J^{\mu}\left(f_{1}^{-1}(C), f_{2}^{-1}(C)\right) \\
& \quad=a\left(E_{C}\right)-J^{\mu}\left(f_{1}^{-1}(C), g^{-1}(C)\right)-J^{\mu}\left(f_{2}^{-1}(C), f_{1}^{-1}(C)\right) \\
& \quad=a\left(E_{C}\right)-J^{\mu}\left(f_{2}^{-1}(C), g^{-1}(C)\right)=\widetilde{a}\left(f_{2}, g ; C\right), \\
& \widetilde{a}\left(f, g_{1} ; C\right)-J^{\mu}\left(g_{1}^{-1}(C), g_{2}^{-1}(C)\right) \\
& \quad=a\left(E_{C}\right)-J^{\mu}\left(f^{-1}(C), g_{1}^{-1}(C)\right)-J^{\mu}\left(g_{1}^{-1}(C), g_{2}^{-1}(C)\right) \\
& \quad=a\left(E_{C}\right)-J^{\mu}\left(f^{-1}(C), g_{2}^{-1}(C)\right)=\widetilde{a}\left(f, g_{2} ; C\right) .
\end{aligned}
$$


$\left(*_{3}\right)$ Let $(K, L) \in \mathcal{N}^{(2)}(M), A \in \mathcal{C}\left(K^{c}\right)$ and $f, g \in \mathcal{D}$. Set $N=A \cap L$ and $\mathcal{C}\left(A \cap L^{c}\right)=\left\{B_{1}, \cdots, B_{m}\right\}$. Note that (a) $a\left(E_{N}\right)=0$ since $N$ is compact and $E_{N}=\emptyset$ and (b) $a\left(E_{A}\right)=\sum_{i} a\left(E_{B_{i}}\right)$ since $E_{A}$ is the disjoint union of $E_{B_{i}}(i=1, \cdots, m)$. Thus by Lemma 2.1(3) we have

$$
\begin{gathered}
\widetilde{a}(f, g ; N)+\sum_{i} \widetilde{a}\left(f, g ; B_{i}\right) \\
=-J^{\mu}\left(f^{-1}(N), g^{-1}(N)\right)+\sum_{i}\left(a\left(E_{B_{i}}\right)-J^{\mu}\left(f^{-1}\left(B_{i}\right), g^{-1}\left(B_{i}\right)\right)\right) \\
=a\left(E_{A}\right)-J^{\mu}\left(f^{-1}(A), g^{-1}(A)\right)=\widetilde{a}(f, g ; A) . \\
\left(*_{4}\right) \widetilde{a}\left(i d_{M}, i d_{M} ; M\right)=a\left(E_{M}\right)-J^{\mu}(M, M)=0 .
\end{gathered}
$$

Proof of Theorem 1.2. Theorem 3.1 yields an admissible sequence $\left(K_{k}, L_{k}\right.$, $\left.f^{k}, g^{k}\right)_{k \geq 1}$ and the limit maps $f, g: P \rightarrow \mathcal{D}_{\partial}(M)_{1}$ which satisfy the following conditions:

(1) $\widetilde{a}(f, g ; C)=0 \quad$ for any $C \in \mathcal{C}\left(\operatorname{cl}\left(K_{k}-L_{k-1}\right)\right) \cup \mathcal{C}\left(\operatorname{cl}\left(L_{k}-K_{k}\right)\right) \cup \mathcal{C}\left(K_{k}^{c}\right) \cup$ $\mathcal{C}\left(L_{k}^{c}\right) \quad(k \geq 1)$

(2) If $p \in P$ and $\widetilde{a}_{p}\left(i d_{M}, i d_{M} ; C\right)=0(C \in \mathcal{F})$, then $f_{p}=g_{p}=i d_{M}$.

For any $C \in \mathcal{C}\left(\operatorname{cl}\left(K_{k}-L_{k-1}\right)\right) \cup \mathcal{C}\left(\operatorname{cl}\left(L_{k}-K_{k}\right)\right)(k \geq 1)$, since $C$ is compact, it follows that $E_{C}=\emptyset$ and $\mu\left(f^{-1}(C)\right), \mu\left(g^{-1}(C)\right)<\infty$, so that

$$
0=\widetilde{a}(f, g ; C)=-J^{\mu}\left(f^{-1}(C), g^{-1}(C)\right)=\left(g_{*} \mu\right)(C)-\left(f_{*} \mu\right)(C) .
$$

Thus, by Lemma 3.5 we obtain a map $\chi: P \rightarrow \mathcal{D}_{\partial}(M)_{1}$ such that

(3) (i) $\chi_{*}\left(f_{*} \mu\right)=g_{*} \mu$,

(ii) if $p \in P$ and $\left(f_{p}\right)_{*} \mu_{p}=\left(g_{p}\right)_{*} \mu_{p}$, then $\chi_{p}=i d_{M}$,

(iii) $\chi(C)=C\left(C \in \mathcal{C}\left(\operatorname{cl}\left(K_{k}-L_{k-1}\right)\right) \cup \mathcal{C}\left(\operatorname{cl}\left(L_{k}-K_{k}\right)\right), k \geq 1\right)$.

We show that the map $h: P \rightarrow \mathcal{D}_{\partial}(M)_{1}, h_{p}=g_{p}^{-1} \chi_{p} f_{p}(p \in P)$ satisfies the following required conditions:

(a) By Remark 4.2 we have $h_{p} \in \mathcal{D}_{\partial}(M)_{1} \cap \mathcal{D}_{\partial}\left(M ; \mu_{p}\right)=\mathcal{D}_{\partial}\left(M ; \mu_{p}\right)_{1}$.

(b) For any $A \in \mathcal{C}\left(K_{k}^{c}\right)(k \geq 1)$, since $f_{p} \in \mathcal{D}_{E_{M}}(M)(p \in P)$, we have $E_{A}=$ $E_{f^{-1}(A)}$, and by condition (1) it follows that

$$
c_{h}^{\mu}\left(E_{A}\right)=c_{h}^{\mu}\left(E_{f^{-1}(A)}\right)=J^{\mu}\left(f^{-1}(A), h f^{-1}(A)\right)=J^{\mu}\left(f^{-1}(A), g^{-1}(A)\right)=a\left(E_{A}\right) .
$$

In general, for any $F \in \mathcal{Q}\left(E_{M}\right)$, there exists $k \geq 1$ and $A_{1}, \cdots, A_{\ell} \in$ $\mathcal{C}\left(K_{k}^{c}\right)$ such that $F=\bigcup_{i} E_{A_{i}}$ (a disjoint union). Then, we have $c_{h}^{\mu}(F)=$ $\sum_{i} c_{h}^{\mu}\left(E_{A_{i}}\right)=\sum_{i} a\left(E_{A_{i}}\right)=a(F)$. This implies that $c_{h}^{\mu}=a$.

(c) If $a_{p}=0$, then $\widetilde{a}_{p}\left(i d_{M}, i d_{M} ; C\right)=0(C \in \mathcal{F})$. Thus $f_{p}=g_{p}=i d_{M}$ by condition (2), and so $\chi_{p}=i d_{M}$ by condition (3) (ii). This implies that $h_{p}=i d_{M}$.

This completes the proof.

Theorem 1.2 has the following slight generalization.

Theorem 1.2' Suppose $P$ is any topological space and $\mu: P \rightarrow \mathcal{V}^{+}(M)$ and $a: P \rightarrow \mathcal{S}(M)$ are continuous maps such that $a_{p} \in \mathcal{S}\left(M ; \mu_{p}\right)(p \in P)$. Then there exists a homotopy $h: P \times[0,1] \rightarrow \mathcal{D}_{\partial}(M)_{1}$ such that for each $(p, t) \in P \times[0,1]$,

(1) $h_{p}(t) \in \mathcal{D}_{\partial}\left(M ; \mu_{p}\right)_{1}$,

(2) $h_{p}(0)=i d_{M}$

(3) $c_{h_{p}(t)}^{\mu_{p}}=t a_{p}$

(4) if $a_{p}=0$, then $h_{p}(t)=i d_{M}$. 
Proof. The homotopy $h$ is obtained by applying Theorem 1.2 to the maps $\bar{\mu}: P \times[0,1] \rightarrow \mathcal{V}^{+}(M), \bar{\mu}_{(p, t)}=\mu_{p} \quad$ and $\quad \bar{a}: P \times[0,1] \rightarrow \mathcal{S}(M), \bar{a}_{(p, t)}=t a_{p}$.

Proof of Corollary 1.2(1). The required section is obtained by applying Theorem 1.2 to the data: $P=\mathcal{S}(M ; \omega)$, the constant map $\mu \equiv \omega: P \rightarrow \mathcal{V}^{+}(M)$ and id $: P \rightarrow \mathcal{S}(M ; \omega)$.

Suppose $G$ is any subgroup of $\mathcal{D}_{E_{M}}(M ; \omega)$ with $\mathcal{D}_{\partial}(M ; \omega)_{1} \subset G$. Consider the restriction $\left.c^{\omega}\right|_{G}: G \rightarrow S(M ; \omega)$. Corollary $1.2(2)$ is a special case of the next corollary.

Corollary 4.2. (1) $\left(G,\left.\operatorname{ker} c^{\omega}\right|_{G}\right) \cong(\mathcal{S}(M ; \omega), 0) \times\left.\operatorname{ker} c^{\omega}\right|_{G}$.

(2) $\left.\operatorname{ker} c^{\omega}\right|_{G}$ is a strong deformation retract of $G$.

Proof. (1) The required homeomorphism $\varphi: G \rightarrow \mathcal{S}(M ; \omega) \times\left.\operatorname{ker} c^{\omega}\right|_{G}$ is defined by $\varphi(h)=\left(c_{h}^{\omega},\left(s\left(c_{h}^{\omega}\right)\right)^{-1} h\right)$. The inverse is given by $\varphi^{-1}(a, f)=s(a) f$.

(2) Since the topological vector space $\mathcal{S}(M ; \omega)$ strong deformation retracts onto $\{0\}$, the conclusion follows from (1).

\section{APPENDIX}

This Appendix includes the proofs of Theorem 2.1 and Lemma 2.3 stated in Section 2.4. These are appropriate parametrized versions of Moser's theorem [9, Theorem 2] and [10, Lemma A2], and we trace the arguments in [9, 10] together with the continuity of related maps with respect to volume forms.

Proof of Theorem 2.1. For simplicity, let $E_{t}=E[0, t](=\partial M \times[0, t])$ and $M_{t}=$ $M-\partial M \times[0, t)$ for $t \in[0,1]$. Consider the subspace of $\mathcal{V}^{+}(M) \times \mathcal{V}^{+}(M) \times(0,1 / 2)$ defined by

$\mathcal{W}^{+}(M)=\left\{(\sigma, \tau, \delta) \in \mathcal{V}^{+}(M) \times \mathcal{V}^{+}(M) \times(0,1 / 2) \mid \sigma(M)=\tau(M), \sigma=\tau\right.$ on $\left.E_{2 \delta}\right\}$.

We show that there exists a continuous map $\psi: \mathcal{W}^{+}(M) \rightarrow \mathcal{D}_{\partial}(M)_{1}$ such that $\psi=\psi(\sigma, \tau, \delta)$ satisfies the following conditions:

(1) $\psi_{*} \sigma=\tau$,

(2) $\psi=i d_{M}$ on $E_{\delta}$,

(3) if $\sigma=\tau$, then $\psi=i d_{M}$.

Then the required map $\varphi: P \rightarrow \mathcal{D}_{\partial}(M)_{1}$ is defined by

$$
\varphi(p)=\psi(\mu(p), \nu(p), \varepsilon(p))(p \in P) .
$$

A construction of the map $\psi$ is described in [9] and [6, Ch I $\S 4]$. As for notation, for a smooth orientable $C^{\infty} n$-manifold $N$, possibly with corners, and a compact subset $C$ of $N$, let

$$
\Omega^{k}(N ; C)=\left\{\lambda \in \Omega^{k}(N) \mid \operatorname{supp} \lambda \subset C\right\} \quad(k \geq 0)
$$

and

$$
\Omega^{n}(N ; C)_{0}=\left\{\omega \in \Omega^{n}(N ; C) \mid \int_{N} \omega=0\right\} .
$$

The arguments in [6. Ch. I §4] (The Poincaré Lemma for compactly supported cohomology) yields a continuous map

$$
\eta_{n}: \Omega^{n}\left(\mathbb{R}^{n} ;[-1,1]^{n}\right)_{0} \rightarrow \Omega^{n-1}\left(\mathbb{R}^{n} ;[-1,1]^{n}\right)
$$


such that $d \eta_{n}(\omega)=\omega \quad\left(\omega \in \Omega^{n}\left(\mathbb{R}^{n} ;[-1,1]^{n}\right)_{0}\right)$. In fact, for $\omega \in \Omega^{n}\left(\mathbb{R}^{n} ;[-1,1]^{n}\right)_{0}$ we have

$$
\omega=(-1)^{n-1} d K \omega+\left(\pi_{*} \omega\right) \wedge e,
$$

where $K \omega \in \Omega^{n-1}\left(\mathbb{R}^{n} ;[-1,1]^{n}\right)$ and $\pi_{*} \omega \in \Omega^{n-1}\left(\mathbb{R}^{n-1} ;[-1,1]^{n-1}\right)_{0}$ are defined as follows. We set $(x, t)=\left(\left(x_{1}, \cdots, x_{n-1}\right), x_{n}\right)$. Choose any 1 -form $e=e(t) d t \in$ $\Omega^{1}(\mathbb{R} ;[-1,1])$ such that $\int_{-\infty}^{\infty} e(s) d s=1$ and put

$$
A(t)=\int_{-\infty}^{t} e(s) d s \in C^{\infty}(\mathbb{R}) .
$$

If $\omega=f d x_{1} \wedge \cdots \wedge d x_{n}\left(f \in C^{\infty}\left(\mathbb{R}^{n}\right)\right.$, supp $\left.f \subset[-1,1]^{n}\right)$, then

$$
K \omega=\bar{f} d x_{1} \wedge \cdots \wedge d x_{n-1} \text {, where } \bar{f}(x, t)=\int_{-\infty}^{t} f(x, s) d s-A(t) \int_{-\infty}^{\infty} f(x, s) d s,
$$

and

$$
\pi_{*} \omega=\left(\int_{-\infty}^{\infty} f(x, s) d s\right) d x_{1} \wedge \cdots \wedge d x_{n-1} .
$$

Thus, if the map $\eta_{n-1}$ is already defined, then $\pi_{*} \omega=d \eta_{n-1}\left(\pi_{*} \omega\right)$ and the map $\eta_{n}$ is recursively defined by

$$
\eta_{n}(\omega)=(-1)^{n-1} K \omega+\eta_{n-1}\left(\pi_{*} \omega\right) \wedge e .
$$

To uniformize the local statement in the preceding paragraph, the Mayer-Vietoris argument is applied by using a good cover and a partition of unity (cf. 6, Ch. I $\S 5])$. Take any open cover $\mathcal{U}=\left\{U_{j}\right\}_{j=1}^{m}$ of $M_{1 / 2}$ in Int $M_{1 / 3}$ such that $\mathcal{U}$ is a good cover of $\bigcup_{j} U_{j}$ and $U_{j} \cap M_{1 / 2} \neq \emptyset(j=1, \cdots, m)$. Then, by the proof of [9, Lemma 1 ], we can find compact subsets $K_{j}$ in $U_{j}(j=1, \cdots, m)$ and continuous maps

$$
\Omega^{n}\left(M ; M_{1 / 2}\right)_{0} \ni \omega \longmapsto \omega_{j} \in \Omega^{n}\left(M ; K_{j}\right)_{0} \quad(j=1, \cdots, m)
$$

such that $\omega=\sum_{j=1}^{m} \omega_{j}$. Since there exists a diffeomorphism from $U_{j}$ onto $\mathbb{R}^{n}$ which maps $K_{j}$ into $[-1,1]^{n}$, we can apply the result in the preceding paragraph to each $U_{j}$ and sum up to obtain a continuous map

$$
\eta: \Omega^{n}\left(M ; M_{1 / 2}\right)_{0} \rightarrow \Omega^{n-1}\left(M ; M_{1 / 3}\right)
$$

such that $d \eta(\omega)=\omega\left(\omega \in \Omega^{n}\left(M ; M_{1 / 2}\right)_{0}\right)$. Replacing $\eta(\omega)$ by $\eta(\omega)-\eta(0)$, we may assume that $\eta(0)=0$.

Now we can apply the Moser's argument [9, Theorem 2]. Define the subspace $\mathcal{W}^{+}(M)_{1 / 4}$ of $\mathcal{V}^{+}(M) \times \mathcal{V}^{+}(M)$ by

$$
\mathcal{W}^{+}(M)_{1 / 4}=\left\{(\sigma, \tau) \in \mathcal{V}^{+}(M) \times \mathcal{V}^{+}(M) \mid \sigma(M)=\tau(M), \sigma=\tau \text { on } E_{1 / 2}\right\}
$$

For each $(\sigma, \tau) \in \mathcal{W}^{+}(M)_{1 / 4}$ we have a smooth family of volume forms $\tau_{t}=\tau+$ $t(\sigma-\tau) \in \mathcal{V}^{+}(M)(t \in[0,1])$. Since $\sigma-\tau \in \Omega^{n}\left(M ; M_{1 / 2}\right)_{0}$, it follows that $\dot{\tau}_{t}=\sigma-\tau=d \eta(\sigma-\tau)$. The linear equation

$$
\iota\left(X_{t}\right) \tau_{t}=-\eta(\sigma-\tau)
$$

uniquely determines a smooth family of vector fields $X_{t}(t \in[0,1])$ on $M$, which induces the associated smooth family of diffeomorphisms $\psi_{t}=\psi_{t}(\sigma, \tau) \in \mathcal{D}(M)$ 
such that $\psi_{0}=i d_{M}$. Equation $(*)$ implies that

(a) $\frac{d}{d t} \psi_{t}^{*} \tau_{t}=\psi_{t}^{*}\left(\dot{\tau}_{t}+\iota\left(X_{t}\right) d \tau_{t}+d \iota\left(X_{t}\right) \tau_{t}\right)=0$; hence $\psi_{t}^{*} \tau_{t}=\tau_{0}$ and $\psi_{1}^{*} \sigma=\tau$,

(b) if $\sigma=\tau$, then $X_{t} \equiv 0$, and so $\psi_{t} \equiv i d_{M}$.

Since $\eta(\sigma-\tau) \in \Omega^{n-1}\left(M ; M_{1 / 3}\right)$, it follows that $\operatorname{supp} X_{t} \subset M_{1 / 3}$ and hence $\operatorname{supp} \psi_{t} \subset M_{1 / 3}$. The coordinate representation of $X_{t}$ in [9, p. 293] shows that the correspondence $(\sigma, \tau) \mapsto\left(X_{t}\right)_{t \in[0,1]}$ is continuous under the compact-open $C^{\infty_{\text {- }}}$ topology. Thus, the correspondence $(\sigma, \tau) \mapsto\left(\psi_{t}\right)_{t \in[0,1]}$ is also continuous under the compact-open $C^{\infty}$-topology, and the map

$$
\psi^{\prime}: \mathcal{W}^{+}(M)_{1 / 4} \rightarrow \mathcal{D}_{E_{1 / 3}}(M)_{1}, \quad \psi^{\prime}=\psi_{1}(\sigma, \tau)^{-1}
$$

satisfies the conditions:

(c) $\psi_{*}^{\prime} \sigma=\tau$,

(d) if $\sigma=\tau$, then $\psi^{\prime}=i d_{M}$.

Finally we incorporate the $(0,1 / 2)$-factor to $\mathcal{W}^{+}(M)_{1 / 4}$. By a suitable smooth sliding in the collar $E$ we can construct a map

$$
\chi:(0,1 / 2) \rightarrow \mathcal{D}_{\partial \cup M_{1}}(M)_{1}
$$

such that

(e) $\chi_{\delta}=i d$ on $E_{\delta / 2}$,

(f) $\chi_{\delta}\left(E_{\delta}\right)=E_{1 / 3}, \quad \chi_{\delta}\left(E_{2 \delta}\right)=E_{1 / 2}$.

Then, the desired map $\psi: \mathcal{W}^{+}(M) \rightarrow \mathcal{D}_{\partial}(M)_{1}$ is defined by

$$
\psi(\sigma, \tau, \delta)=\chi_{\delta}^{-1} \psi^{\prime}\left(\left(\chi_{\delta}\right)_{*} \sigma,\left(\chi_{\delta}\right)_{*} \tau\right) \chi_{\delta} .
$$

This completes the proof.

Proof of Lemma 2.3. Since the maps $\mu, \nu$ factor through the projections $\pi_{1}, \pi_{2}$ from the product $P_{0}=\mathcal{V}^{+}(M) \times \mathcal{V}^{+}(M)$ onto the 1st and 2nd factor, it suffices to consider the case where $(P, \mu, \nu)=\left(P_{0}, \pi_{1}, \pi_{2}\right)$.

(1) We may assume that $(M, E, S)=(S \times \mathbb{R}, S \times[-1,1], S \times\{0\})$, where $S$ is an oriented $C^{\infty}(n-1)$-manifold, possibly with boundary, and the orientation of $M$ is induced from that of $S$ and the standard orientation of $\mathbb{R}$. We fix $\omega_{0} \in \mathcal{V}^{+}(S)$ and set $\omega=\omega_{0} \wedge d t \in \mathcal{V}^{+}(M)$.

We shall construct maps

$$
\psi: \mathcal{V}^{+}(S) \rightarrow \mathcal{D}_{S \cup\left(M-E_{U}\right)}(M)_{1} \quad \text { and } \quad \delta: \mathcal{V}^{+}(S) \rightarrow C^{\infty}(S,(0,1))
$$

such that for $\mu \in \mathcal{V}^{+}(S)$,

(i) $\left(\psi_{\mu}\right)_{*} \mu=\omega$ on $E_{K}\left[-\delta_{\mu}, \delta_{\mu}\right]$,

(ii) ${ }^{\prime}$ for each $x \in S$,

(a) $\psi_{\mu}\left(E_{x}^{ \pm}\right)=E_{x}^{ \pm}$,

(b) $\left.\left(\psi_{\mu}\right)\right|_{E_{x}^{ \pm}}$depends only on $\left.\mu\right|_{E_{x}^{ \pm}}$(i.e., if $\mu, \nu \in \mathcal{V}^{+}(M)$ and $\mu=\nu$ on $E_{x}^{ \pm}$, then $\psi_{\mu}=\psi_{\nu}$ on $\left.E_{x}^{ \pm}\right)$

Then the required map $\varphi: P_{0} \rightarrow \mathcal{D}_{S \cup\left(M-E_{U}\right)}(M)_{1}$ is defined by $\varphi_{(\mu, \nu)}=\psi_{\nu}^{-1} \psi_{\mu}$. Since

$$
\left(\psi_{\mu}\right)_{*} \mu=\omega=\left(\psi_{\nu}\right)_{*} \nu \quad \text { on } \quad E_{K}\left[-\delta_{(\mu, \nu)}, \delta_{(\mu, \nu)}\right] \quad\left(\delta_{(\mu, \nu)}=\min \left\{\delta_{\mu}, \delta_{\nu}\right\}\right),
$$

if we choose a map $\varepsilon: P_{0} \rightarrow C^{\infty}(S,(0,1))$ so that $\varepsilon_{(\mu, \nu)}$ is sufficiently small for each $(\mu, \nu) \in P_{0}$, then the maps $\varphi$ and $\varepsilon$ satisfy the required conditions. 
The construction of the map $\psi$ is described in [10, Lemma A2]. The key observation is that for each $f \in C^{\infty}(M)$ the associated map

$$
\varphi_{f}: M \rightarrow M, \varphi_{f}(x, t)=(x, f(x, t))
$$

has the property that $\left(\varphi_{f}\right)^{*} \omega=f_{t} \omega$, where $f_{t}=\frac{\partial f}{\partial t}$.

Consider the following subspaces of $C^{\infty}(M)$ :

$$
\begin{gathered}
C^{\infty}(M)_{>0}=\left\{f \in C^{\infty}(M) \mid f>0\right\}, \\
C^{\infty}(M)^{\prime}=\left\{f \in C^{\infty}(M) \mid f(x, 0)=0, f_{t}>0\right\} .
\end{gathered}
$$

Take three functions $\alpha \in C^{\infty}(\mathbb{R},(-1 / 2,1 / 2)), \lambda \in C^{\infty}(\mathbb{R},[0,1])$ and $\rho \in$ $C^{\infty}(S,[0,1])$ such that

(a) $\alpha(t)=t(|t| \leq 1 / 3), \quad \alpha_{t}>0$,

(b) $\lambda(t)=\lambda(-t), \quad \lambda(t)=0(|t| \leq 1 / 2), \quad \lambda(t)=1(|t| \geq 1), \quad \lambda_{t}(t) \geq 0(t \geq 0)$,

(c) $\rho(x)=0(x \in V), \rho(x)=1$ on $(x \in S-U)$, where $V$ is an open subset of $S$ with $K \subset V \subset U$.

Choose a continuous map $\gamma: C^{\infty}(M)^{\prime} \rightarrow C^{\infty}(S,(0,1 / 2))$ such that

$$
f\left(E\left[-\gamma_{f}, \gamma_{f}\right]\right) \subset[-1 / 3,1 / 3] \quad\left(f \in C^{\infty}(M)^{\prime}\right) .
$$

For each $f \in C^{\infty}(M)^{\prime}$ we define $\tilde{f} \in C^{\infty}(M)^{\prime}$ by

$$
\begin{gathered}
\tilde{f}(x, t)=(1-\rho(x)) g(x, t)+\rho(x) \cdot t, \\
g(x, t)=(1-\lambda(t)) \alpha(f(x, t))+\lambda(t) \cdot t \quad((x, t) \in M) .
\end{gathered}
$$

Since $\widetilde{f}(x, t)=t\left((x, t) \in S \cup\left(M-E_{U}\right)\right)$ and $\tilde{f}=f$ on $E_{V}\left[-\gamma_{f}, \gamma_{f}\right]$, it follows that

(a) $\varphi_{\tilde{f}} \in \mathcal{D}_{S \cup\left(M-E_{U}\right)}(M)_{1}$,

(b) $\left(\varphi_{\tilde{f}}\right)^{*} \omega=\widetilde{f}_{t} \omega=f_{t} \omega$ on $E_{V}\left[-\gamma_{f}, \gamma_{f}\right]$.

Each $\mu \in \mathcal{V}^{+}(M)$ uniquely determines $h_{\mu} \in C^{\infty}(M)_{>0}$ such that $\mu=h_{\mu} \omega$. Define $f_{\mu} \in C^{\infty}(M)^{\prime}$ by

$$
f_{\mu}(x, t)=\int_{0}^{t} h_{\mu}(x, s) d s .
$$

Since $\left(f_{\mu}\right)_{t}=h_{\mu}$, it is seen that

$$
\left(\varphi_{\widetilde{f}_{\mu}}\right)^{*} \omega=h_{\mu} \omega=\mu \text { on } E_{V}\left[-\gamma_{f_{\mu}}, \gamma_{f_{\mu}}\right] .
$$

Finally we define the map $\psi: \mathcal{V}^{+}(S) \rightarrow \mathcal{D}_{S \cup\left(M-E_{U}\right)}(M)_{1}$ by $\psi_{\mu}=\varphi_{\widetilde{f_{\mu}}}$. Condition (ii) ${ }^{\prime}$ follows from the construction of the map $\psi$. If we choose a map $\delta: \mathcal{V}^{+}(S) \rightarrow C^{\infty}(S,(0,1))$ so that $\delta_{\mu}$ is sufficiently small for each $\mu \in \mathcal{V}^{+}(S)$, then the maps $\psi$ and $\delta$ satisfy condition (i)'.

(2) We may assume that $(M, E, \partial M)=(S \times[0, \infty), S \times[0,1], S \times\{0\})$, where $S$ is an oriented $C^{\infty}(n-1)$-manifold, possibly with boundary. Assertion (2) follows from the same argument as in (1) by ignoring the $(-\infty, 0]$-side.

\section{REFERENCES}

[1] S. R. Alpern and V.S. Prasad, Typical Dynamics of Volume-Preserving Homeomorphisms, Cambridge Tracts in Mathematics, Cambridge University Press, 2001.

[2] A. Banyaga, Formes-volume sur les variétés à bord, Enseignement Math. (2) 20 (1974) 127 131. MR0358649 (50:11108)

[3] A. Banyaga, The Structure of Classical Diffeomorphism Groups, Mathematics and Its Applications 400, Kluwer Academic Publishers Group, Dordrecht, 1997. MR.1445290 (98h:22024) 
[4] R. Berlanga, Groups of measure-preserving homeomorphisms as deformation retracts, J. London Math. Soc. (2) 68 (2003) 241 - 254. MR1980255 (2004d:57040)

[5] R. Berlanga and D.B.A. Epstein, Measures on sigma-compact manifolds and their equivalence under homeomorphism, J. London Math. Soc. (2) 27 (1983) 63 - 74 . MR686504 (84m:28023)

[6] R. Bott and L. W. Tu, Differential Forms in Algebraic Topology, Graduate Texts in Mathematics 82, Springer-Verlag, New York-Berlin, 1982. MR658304 (83i:57016)

[7] A. Fathi, Structures of the group of homeomorphisms preserving a good measure on a compact manifold, Ann. Scient. Ec. Norm. Sup. (4) 13 (1980) 45 - 93. MR.584082 (81k:58042)

[8] R. E. Greene and K. Shiohama, Diffeomorphisms and volume-preserving embeddings of noncompact manifolds, Trans. Amer. Math. Soc. 255 (1979) 403 - 414. MR.542888 (80k:58031)

[9] J. Moser, On the volume elements on a manifold, Trans. Amer. Math. Soc. 120 (1965) 286 294. MR0182927 (32:409)

[10] D. McDuff, On groups of volume-preserving diffeomorphisms and foliations with transverse volume form, Proc. London Math. Soc. (3) 43 (1981) 295 - 320. MR628279 (83g:58007)

[11] J. Oxtoby and S. Ulam, Measure preserving homeomorphisms and metrical transitivity, Ann. of Math. 42 (1941) 874 - 920. MR0005803(3:211b)

[12] T. Yagasaki, Measure-preserving homeomorphisms of noncompact manifolds and mass flow toward ends, Fund. Math. 197 (2007) 271 - 287. MR2365892 (2009f:57056)

Division of Mathematics, Graduate School of Science and Technology, Kyoto Institute of Technology, Kyoto, 606-8585, Japan

E-mail address: yagasaki@kit.ac.jp 